\title{
Classifying Finite-Dimensional C*-Algebras by Posets of Their Commutative $C^{*}$-Subalgebras
}

\author{
A. J. Lindenhovius ${ }^{1}$
}

Received: 15 October 2014 / Accepted: 22 October 2014 / Published online: 19 October 2015

(C) The Author(s) 2015. This article is published with open access at Springerlink.com

\begin{abstract}
We consider the functor $\mathcal{C}$ that to a unital $\mathrm{C}^{*}$-algebra $A$ assigns the partial order set $\mathcal{C}(A)$ of its commutative $\mathrm{C}^{*}$-subalgebras ordered by inclusion. We investigate how some $\mathrm{C}^{*}$-algebraic properties translate under the action of $\mathcal{C}$ to order-theoretical properties. In particular, we show that $A$ is finite dimensional if and only $\mathcal{C}(A)$ satisfies certain chain conditions. We eventually show that if $A$ and $B$ are $C^{*}$-algebras such that $A$ is finite dimensional and $\mathcal{C}(A)$ and $\mathcal{C}(B)$ are order isomorphic, then $A$ and $B$ must be ${ }^{*}$-isomorphic.
\end{abstract}

Keywords Finite-dimensional $\mathrm{C}^{*}$-algebras $\cdot$ Commutative $\mathrm{C}^{*}$-subalgebras $\cdot$ Order theory $\cdot$ Chain conditions $\cdot$ Directly indecomposable lattices

\section{Introduction}

Given a $\mathrm{C}^{*}$-algebra $A$ with unit $1_{A}$, let $\mathcal{C}(A)$ be the set of all commutative unital $\mathrm{C}^{*}$ subalgebras $C$ of $A$ such that $1_{A} \in C$. Then $\mathcal{C}(A)$ becomes a poset ${ }^{1}$ if we order it by inclusion. Now, one could consider the following question: is it possible to recover the structure of $A$ as a $C^{*}$-algebra from the poset $\mathcal{C}(A)$ ? More precisely, if $A$ and $B$ are $\mathrm{C}^{*}$-algebras such that $\mathcal{C}(A)$ and $\mathcal{C}(B)$ are isomorphic as posets, can we find an $*$-isomorphism between $A$ and $B$ ?

Apart from its mathematical relevance, this problem is of considerable importance for the so called quantum toposophy program, where one tries to describe quantum mechanics in

\footnotetext{
${ }^{1}$ We refer to the appendix for an overview of the necessary concepts in order theory.
}

This article is dedicated to my sister.

A.J. Lindenhovius

aj.lindenhovius@math.ru.nl

1 Department of Mathematics Radboud, University Nijmegen, P.O. Box 9010, 6500 GL, Nijmegen, The Netherlands 
terms of topos theory (see e.g., [6, 9, 23, 41]). In this program, the central objects of research are the topoi $\operatorname{Sets}^{\mathcal{C}(A)}$ and $\operatorname{Sets}{ }^{\mathcal{C}(A)^{\mathrm{op}}}$. The motivation behind at least [23] and [41] is Niels Bohr's doctrine of classical concepts, which, roughly speaking, states that a measurement provides a "classical snapshot of quantum reality". Mathematically, this corresponds with an element of $\mathcal{C}(A)$, and knowledge of all classical snapshots should provide a picture of quantum reality, that is as complete as (humanly) possible. The possiblility of reconstructing $A$ from $\mathcal{C}(A)$ would assure the soundness of this doctrine.

For commutative $C^{*}$-algebras, Mendivil showed that the answer to the question is affirmative (see [32]). It turns out that more can be said about the commutative case. As we will see, $\mathcal{C}$ turns out to be a functor from unital $C^{*}$-algebras to posets. Hence if $f: A \rightarrow B$ is a *-isomorphism, then $\mathcal{C}(f): \mathcal{C}(A) \rightarrow \mathcal{C}(B)$ is an order isomorphism. In [17], Hamhalter gave not only a different proof of Mendivil's statement that an order isomorphism $\psi: \mathcal{C}(A) \rightarrow \mathcal{C}(B)$, with $A$ and $B$ commutative $C^{*}$-algebras, yields an *-isomorphism $f: A \rightarrow B$, but he showed as well that this *-isomorphism can be constructed in such a way that $\psi=\mathcal{C}(f)$. Moreover, he proved that as long as $A$ is not two dimensional, there is only one ${ }^{*}$-isomorphism that induces $\psi$ in this way.

For non-commutative $C^{*}$-algebras however, the answer is negative, since Connes [7] showed the existence of a $C^{*}$-algebra $A_{c}$ (actually even a von Neumann algebra) that is not isomorphic to its opposite algebra $A_{c}^{\mathrm{op}}$. Here the opposite algebra is the $\mathrm{C}^{*}$-algebra with the same underlying topological vector space, but with multiplication defined by $(a, b) \mapsto b a$, where $(a, b) \mapsto a b$ denotes the original multiplication. Since $\mathcal{C}(A)$ is always isomorphic to $\mathcal{C}\left(A^{\text {op }}\right)$ as poset, for each $\mathrm{C}^{*}$-algebra $A$, the existence of Connes' $\mathrm{C}^{*}$-algebra $A_{c}$ shows that the order structure of $\mathcal{C}(A)$ is not always enough in order to reconstruct $A$. More recent counterexamples can be found in [34] and [35].

Nevertheless, there are still problems one could study. For instance, in [10] Döring and Harding consider a functor similar to $\mathcal{C}$, namely the functor $\mathcal{V}$ assigning to a von Neumann algebra $M$ the poset $\mathcal{V}(M)$ of its commutative von Neumann subalgebras, and prove that one can reconstruct the Jordan structure, i.e., the anticommutator $(a, b) \mapsto a b+b a$, of $M$ from $\mathcal{V}(M)$. Similarly, in [17], it is shown that if $\mathcal{C}(A)$ and $\mathcal{C}(B)$ are order isomorphic, then there exists a quasi-linear Jordan isomorphism between $A_{\mathrm{sa}}$ and $B_{\mathrm{sa}}$, the sets of self-adjoint elements of $A$ and $B$, respectively. Here quasi-linear means linear with respect to elements that commute. In [18], it is even shown that this quasi-linear Jordan isomorphism is linear when $A$ and $B$ are $A W^{*}$-algebras.

Moreover, one could replace $\mathcal{C}(A)$ by a structure with stronger properties. An example of such a structure is an active lattice, defined in [25], where Heunen and Reyes also show that this structure is strong enough to determine $\mathrm{AW}^{*}$-algebras completely.

In this paper, however, we will only study $\mathcal{C}$ as a functor and as an invariant for $\mathrm{C}^{*}$-algebras. We shall examine some of its properties, and, as our main result, we shall prove that if $A$ is finite dimensional, $\mathcal{C}(A)$ has enough structure to determine $A$ up to *-isomorphism. The way this result is proven considerably differs from the methods of Hamhalter and Mendivil in the commutative case, since we cannot use Gel'fand duality and the ensuing topological methods as they do. Our approach also differs from Hamhalter's methodes in the sense that if $\psi: \mathcal{C}(A) \rightarrow \mathcal{C}(B)$ is an order isomorphism with $A$ finite dimensional, we do not construct a *-isomorphism $f: A \rightarrow B$ such that $\mathcal{C}(f)=\psi$, since it will turn out that this is not always possible. Instead, we look at order-theoretical invariants in $\mathcal{C}(A)$ that reflect the $\mathrm{C}^{*}$-algebraic invariants in $A$ determinining $A$ up to $*$-isomorphism.

The first step is to find order-theoretical properties of $\mathcal{C}(A)$ that corresponds to $A$ being finite dimensional. These properties turn out to be chain conditions on $\mathcal{C}(A)$. The next step is to identify $Z(A)$, the center of $A$, as element of $\mathcal{C}(A)$. This turns out to be the 
infimum of all maximal elements of $\mathcal{C}(A)$. Finally, we consider the interval $[Z(A), M]$ in $\mathcal{C}(A)$, where $M$ is any maximal element. This interval is a lattice, which factors into a product of directly indecomposable lattices. The length of the maximal chains in these lattices corresponds exactly to the dimensions of the matrix algebra factors of $A$, since by the Artin-Wedderburn Theorem $A$ factors in a unique way into a product of matrix algebras.

\section{$2 C^{*}$-Subalgebras of a Commutative $C^{*}$-Algebra}

All $\mathrm{C}^{*}$-algebras in this article are assumed to be unital.

Definition 1 Let $A$ be a $C^{*}$-subalgebra of $C(X)$. Then we define an equivalence relation $\sim_{A}$ on $X$ by $x \sim_{A} y$ if and only if $f(x)=f(y)$ for each $f \in A$. We denote the equivalence class of $x$ under this equivalence relation by $[x]_{A}$.

Lemma 1 [40, Proposition 5.1.3] A $C^{*}$-algebra $A$ is a $C^{*}$-subalgebra of $C(X)$ if and only if there is a compact Hausdorff space $Y$ and a continuous surjection $q: X \rightarrow Y$ such that $C_{q}[C(Y)]=A$. The map $C_{q}: C(Y) \rightarrow C(X), f \mapsto f \circ q$ is an isometric *-homomorphism in this case, and $Y$ and $X / \sim_{A}$ are homeomorphic.

Definition 2 Let $X$ be compact Hausdorff and let $K \subseteq X$ be closed. Then we denote the $\mathrm{C}^{*}$-subalgebra $\{f \in C(X): f$ is constant on $K\}$ by $C_{K}$.

Lemma 2 [17, Proposition 2.2] Let $A$ be a $C^{*}$-subalgebra of $C(X)$. Then

$$
A=\bigcap_{x \in X} C_{[x]_{A}},
$$

where $[x]_{A}$ is interpreted as a subset of $X$ (rather than as a point in $\left.X / \sim_{A}\right)$.

Proof By Lemma 1, we have $A=C_{q}[C(Y)]$ with $Y=X / \sim_{A}$ and $q: X \rightarrow Y$ the quotient map. So each $f \in A$ is of the form $g \circ q$ for some $g \in C(Y)$, meaning that $f$ is constant on $[x]_{A}$ for each $x \in X$. Conversely, let $f$ be constant on $[x]_{A}$ for each $x \in X$. Define $g: Y \rightarrow X$ by $g\left([x]_{A}\right)=f(x)$. Then $g$ is well defined, since $f$ is constant on $[x]_{A}$, and $f=g \circ q$. Moreover, let $U \subseteq \mathbb{C}$ be open. Then $g^{-1}[U]$ is open in $Y$ if and only if $q^{-1}\left[g^{-1}[U]\right]$ is open in $X$ by definition of the quotient topology. Since $f=g \circ q$, we find $g^{-1}[U]$ is open in $Y$ if and only if $f^{-1}[U]$ is open in $X$. Since $f$ is continuous, we find that $g^{-1}[U]$ is open, so $g \in C(X)$. Hence $f$ lifts to a function on $C(Y)$, so $f \in A$. Thus

$$
\begin{aligned}
A & =\left\{f \in C(X): f \text { is constant on }[x]_{A} \forall x \in X\right\} \\
& =\bigcap_{x \in X}\left\{f \in C(X): f \text { is constant on }[x]_{A}\right\} \\
& =\bigcap_{x \in X} C_{[x]_{A}} .
\end{aligned}
$$




\section{The Functor $\mathcal{C}$}

Definition 3 Let $A$ be a $C^{*}$-algebra with unit $1_{A}$. We denote the set of its commutative $\mathrm{C}^{*}$-subalgebras containing $1_{A}$ by $\mathcal{C}(A)$.

If we denote the category of unital $\mathrm{C}^{*}$-algebras with unital *-homomorphisms as morphisms by $\mathbf{u C S t a r}$ and the category of posets with order morphisms as morphisms by Poset, then $\mathcal{C}: \mathbf{u C S t a r} \rightarrow$ Poset can be made into a functor [21, Proposition 5.3.3].

Lemma $3 \mathcal{C}:$ uCStar $\rightarrow$ Poset becomes a functor if for $*$-homomorphisms $f: A \rightarrow B$ between $C^{*}$-algebras $A$ and $B$ we define $\mathcal{C}(f): \mathcal{C}(A) \rightarrow \mathcal{C}(B)$ by $C \mapsto f[C]$.

Proof Let $C \in \mathcal{C}(A)$. Then the restriction of $f$ to $C$ is a *-homomorphism with codomain $B$. It follows from the First Isomorphism Theorem for $\mathrm{C}^{*}$-algebras (see for instance [33, Theorem 3.1.6]) that $f[C]$ is a $C^{*}$-subalgebra of $B$. Since $f$ is multiplicative, it follows that $f[C]$ is commutative. Clearly $f[C]$ is a *-subalgebra of $B$, so $f[C] \in \mathcal{C}(B)$. Moreover, we have $f[C] \subseteq f[D]$ if $C \subseteq D$, so $\mathcal{C}(f)$ is an order morphism. If $f: A \rightarrow B$ and $g: B \rightarrow D$ are *-homomorphisms, then $\mathcal{C}(g \circ f)(C)=g \circ f[C]=g[f[C]]=\mathcal{C}(g) \circ \mathcal{C}(f)$, and if $I_{A}: A \rightarrow A$ is the identity morphism, then $\mathcal{C}\left(I_{A}\right)=1_{\mathcal{C}(A)}$, the identity morphism of $\mathcal{C}(A)$. Thus $\mathcal{C}$ is indeed a functor.

Lemma 4 Let $A$ be a $C^{*}$-algebra. Then $\mathcal{C}(A)$ has all non-empty infima, where the infimum is given by the intersection operator. In particular, $\mathcal{C}(A)$ is a meet-semilattice, and has a least element $\mathbb{C} 1_{A}=\left\{\lambda 1_{A}: \lambda \in \mathbb{C}\right\}$.

Proof Elementary.

Lemma 5 [3, Proposition 14] Let A be a $C^{*}$-algebra. Then the following statements are equivalent.

(i) A is commutative;

(ii) $\mathcal{C}(A)$ is bounded;

(iii) $\mathcal{C}(A)$ is a complete lattice.

Proof This follows immediately from the observation that $A \in \mathcal{C}(A)$ if and only if $A$ is commutative, and the fact that if $\mathcal{C}(A)$ has a greatest element, it has all infima, so it must be a complete lattice.

The next proposition is originally due to Spitters [38]. A similar statement for the functor $\mathcal{V}$ assigning to a von Neumann algebra $M$ the poset $\mathcal{V}(M)$ of its commutative von Neumann subalgebras can be found in [11].

Proposition 1 Let $A$ be a $C^{*}$-algebra. Then $\mathcal{C}(A)$ is a dcpo, where $\bigvee \mathcal{D}=\overline{\cup \mathcal{D}}$ for each directed $\mathcal{D} \subseteq \mathcal{C}(A)$, and where $\bigvee \mathcal{D}=\bigcup \mathcal{D}$ if $A$ is finite dimensional.

Proof Let $\mathcal{D} \subseteq \mathcal{C}(A)$ be a directed subset. Let $S=\bigcup \mathcal{D}$. We show that $S$ is a commutative *-algebra. Let $x, y \in S$ and $\lambda, \mu \in \mathbb{C}$, there are $D_{1}, D_{2} \in \mathcal{D}$ such that $x \in D_{1}$ and $y \in D_{2}$. 
Since $\mathcal{D}$ is directed, there is some $D_{3} \in \mathcal{D}$ such that $D_{1}, D_{2} \subseteq D_{3}$. Hence $x, y \in D_{3}$, whence $\lambda x+\mu y, x^{*}, x y \in D_{3}$, and since $D_{3}$ is commutative, it follows that $x y=y x$. Since $D_{3} \subseteq S$, it follows that $S$ is a commutative *-subalgebra of $A$. Now, $\bar{S}$ is a commutative $\mathrm{C}^{*}$ subalgebra of $A$, which is the smallest commutative $C^{*}$-subalgebra of $A$ containing every element of $\mathcal{D}$, hence it is the supremum of $\mathcal{D}$. If $A$ is finite dimensional, all subspaces of $A$ are closed, hence $\bigvee \mathcal{D}=\overline{\cup \mathcal{D}}=\bigcup \mathcal{D}$.

If $A$ is not finite dimensional, we do not necessarily have $\bigvee \mathcal{D}=\bigcup \mathcal{D}$ for each directed $\mathcal{D} \subseteq \mathcal{C}(A)$. For instance, let $A=C([0,1])$ and

$$
\mathcal{D}=\left\{C_{[0,1 / n]}: n \in \mathbb{N}\right\} .
$$

Then $\bigcup \mathcal{D}$ consists of functions in $C([0,1])$ that are all constant on some neighborhood of 0 . Hence $f \notin \bigcup \mathcal{D}$, where $f:[0,1] \rightarrow \mathbb{C}$ is defined by $f(x)=x$. However, one can easily show that $\bigcup \mathcal{D}$ is a unital *-subalgebra of $C([0,1])$ that separates all points of $[0,1]$, so $\overline{\cup \mathcal{D}}=C([0,1])$ by the Stone-Weierstrass Theorem. Thus $\bigcup \mathcal{D} \neq \bigvee \mathcal{D}$.

Proposition 2 Let $f: A \rightarrow B$ be $a$ *-homomorphism. Then

(i) If $\mathcal{C}(f)$ is surjective, then $f$ is surjective;

(ii) If $f$ is injective, then $\mathcal{C}(f)$ is an order embedding such that

$$
\downarrow \mathcal{C}(f)[\mathcal{C}(A)]=\mathcal{C}(f)[\mathcal{C}(A)]
$$

and

$$
\mathcal{C}(f)\left(\bigcap_{i \in I} C_{i}\right)=\bigcap_{i \in I} \mathcal{C}(f)\left(C_{i}\right)
$$

for each non-empty family $\left\{C_{i}\right\}_{i \in I} \subseteq \mathcal{C}(A)$. Moreover $\mathcal{C}(f)$ has an upper adjoint $\mathcal{C}(f)_{*}: \mathcal{C}(B) \rightarrow \mathcal{C}(A)$ given by $D \mapsto f^{-1}[D]$ such that

$$
\mathcal{C}(f)_{*} \circ \mathcal{C}(f)=1_{\mathcal{C}(A)}
$$

(iii) If $f$ is a ${ }^{*}$-isomorphism, then $\mathcal{C}(f)$ is an order isomorphism.

Proof

(i) Assume that $\mathcal{C}(f)$ is surjective. Let $b \in B$, and write $b=\lambda_{1} b_{1}+\lambda_{2} b_{2}$, where $b_{1}, b_{2} \in$ $B$ self-adjoint and $\lambda_{1}, \lambda_{2} \in \mathbb{C}$. Then $C^{*}\left(b_{i}, 1_{B}\right) \in \mathcal{C}(B)$ for each $i=1,2$, hence by the surjectivity of $\mathcal{C}(f)$, there are $C_{1}, C_{2} \in \mathcal{C}(A)$ such that $\mathcal{C}(f)\left(C_{i}\right)=C^{*}\left(b_{i}, 1_{B}\right)$. Since $\mathcal{C}(f)\left(C_{i}\right)=f\left[C_{i}\right]$, this means that there are $a_{1} \in C_{1}$ and $a_{2} \in C_{2}$ such that $f\left(a_{i}\right)=b_{i}$. Let $a=\lambda_{1} a_{1}+\lambda a_{2}$. Then $f(a)=b$, hence $f$ is surjective.

(ii) Assume that $f$ is injective. We first show that $\mathcal{C}(f)$ has an upper adjoint $\mathcal{C}(f)_{*}$. Let $D \in \mathcal{C}(B)$ and $x, y \in f^{-1}[D]$. Then $f(x), f(y) \in D$, so

$$
f(x y-y x)=f(x) f(y)-f(y) f(x)=0 .
$$

By the injectivity of $f$ it follows that $x y=y x$, so $f^{-1}[D]$ is a commutative *subalgebra of $A$, which is closed since $f$ is continuous and $D$ is closed. Moreover, since $f\left(1_{A}\right)=1_{B}$, and $1_{B} \in D$, it follows that $1_{A} \in f^{-1}[D]$. So $D \mapsto f^{-1}[D]$ is a well-defined map $\mathcal{C}(B) \rightarrow \mathcal{C}(A)$. Moreover, this map is clearly inclusion preserving, and since $f[C] \subseteq D$ if and only if $C \subseteq f^{-1}[D]$ for any two subsets $C$ and $D$ of $A$ and $B$, respectively, we find that $D \mapsto f^{-1}[D]$ is indeed the upper adjoint of $\mathcal{C}(f)$. 
Let $\left\{C_{i}\right\}_{i \in I}$ be a non-empty collection of elements of $\mathcal{C}(A)$. We always have $f\left[\bigcap_{i \in I} C_{i}\right] \subseteq \bigcap_{i \in I} f\left[C_{i}\right]$. Now, let $x \in \bigcap_{i \in I} f\left[C_{i}\right]$. Then for each $i \in I$ there is an $c_{i} \in C_{i}$ such that $x=f\left(c_{i}\right)$. Hence for each $i, j \in I$, we have $f\left(c_{i}\right)=f\left(c_{j}\right)$. By injectivity of $f$ it follows that $c_{i}=c_{j}$, so $x=f(c)$ with $c \in \bigcap_{i \in I} C_{i}$ equal to $c_{i}$ for each $i \in I$. We conclude that $f\left[\bigcap_{i \in I} C_{i}\right]=\bigcap_{i \in I} f\left[C_{i}\right]$, which is exactly (1).

By injectivity of $f$, we find

$$
\mathcal{C}(f)_{*} \circ \mathcal{C}(f)(C)=f^{-1}[f[C]]=C
$$

for each $C \in \mathcal{C}(A)$, hence $\mathcal{C}(f)_{*} \circ \mathcal{C}(f)=1_{\mathcal{C}(A)}$.

Let $C \in \downarrow \mathcal{C}(f)[\mathcal{C}(A)]$. Hence there is some $D \in \mathcal{C}(A)$ such that $C \subseteq \mathcal{C}(f)(D)$.

Then

$$
\mathcal{C}(f)_{*}(C) \subseteq \mathcal{C}(f)_{*} \circ \mathcal{C}(f)(D)=D
$$

Now,

$$
\mathcal{C}(f) \circ \mathcal{C}(f)_{*}(C)=f\left[f^{-1}[C]\right]=C \cap f[A]=C,
$$

since $C \subseteq \mathcal{C}(f)(D)=f[D] \subseteq f[A]$. So $C=\mathcal{C}(f)(E)$ with $E=\mathcal{C}(f)_{*}(C)$, hence $C \in \mathcal{C}(f)[\mathcal{C}(A)]$. We conclude that $\downarrow \mathcal{C}(f)[\mathcal{C}(A)]=\mathcal{C}(f)[\mathcal{C}(A)]$.

Finally, we show that $\mathcal{C}(f)$ is an order embedding. Let $C_{1}, C_{2} \in \mathcal{C}(A)$. Since $\mathcal{C}(f)$ is an order morphism, we have $\mathcal{C}(f)\left(C_{1}\right) \subseteq \mathcal{C}(f)\left(C_{2}\right)$ if $C_{1} \subseteq C_{2}$. Assume now that $\mathcal{C}(f)\left(C_{1}\right) \subseteq \mathcal{C}(f)\left(C_{2}\right)$. In other words, we have $f\left[C_{1}\right] \subseteq f\left[C_{2}\right]$. By the injectivity of $f$, we have $f^{-1}[f[C]]=C$ for each $C \in \mathcal{C}(A)$. Since $f^{-1}$ preserves inclusions, this implies $C_{1} \subseteq C_{2}$. Hence we obtain $\mathcal{C}(f)\left(C_{1}\right) \subseteq \mathcal{C}(f)\left(C_{2}\right)$ if and only if $C_{1} \subseteq C_{2}$. In other words, $\mathcal{C}(f)$ is an order embedding.

(iii) This follows directly from the functoriality of $\mathcal{C}$ and the fact that $f$ has an inverse.

\section{Finite-Dimensional $C^{*}$-Algebras}

In this section we shall prove the following theorem.

Theorem 1 Let $A$ be a finite-dimensional $C^{*}$-algebra and $B$ any $C^{*}$-algebra such that $\mathcal{C}(A) \cong \mathcal{C}(B)$. Then $A \cong B$.

The proof of the theorem relies on the fact that every finite-dimensional $\mathrm{C} *$-algebra is *-isomorphic to a finite sum of matrix algebras.

Theorem 2 (Artin-Wedderburn) Let $A$ be a finite-dimensional $C^{*}$-algebra. Then there are $k, n_{1}, \ldots, n_{k} \in \mathbb{N}$ such that

$$
A \cong \bigoplus_{i=1}^{k} \mathrm{M}_{n_{i}}(\mathbb{C}) .
$$

The number $k$ is unique, whereas the numbers $n_{1}, \ldots, n_{k}$ are unique up to permutation. 
Proof [39, Theorem I.11.2]

The strategy for proving Theorem 1 is the following. First, we find an order-theoretic property of $\mathcal{C}(A)$ that corresponds with the finite-dimensionality of $A$. Then we find a method of retrieving the numbers $k, n_{1}, \ldots, n_{k}$ from $\mathcal{C}(A)$.

Definition 4 Let $\mathcal{C}$ be a poset. Then $\mathcal{C}$ is called Artinian if it satisfies one of the following equivalent conditions:

1) Every non-empty subset of $\mathcal{C}$ contains a minimal element;

2) All non-empty filtered subsets of $\mathcal{C}$ have a least element;

3) $\mathcal{C}$ satisfies the descending chain condition: if we have a sequence of elements $C_{1} \geq$ $C_{2} \geq \ldots$ in $\mathcal{C}$, i.e., a countable descending chain, then the sequence stabilizes, i.e., there is an $n \in \mathbb{N}$ such that $C_{k}=C_{n}$ for all $k>n$.

In order to see that these conditions are equivalent, assume that (1) holds and let $\mathcal{F}$ a nonempty filtered subset of $\mathcal{C}$. Then $\mathcal{F}$ must have a minimal element $C$. Now, if $F \in \mathcal{F}$, then there must be an $G \in \mathcal{F}$ such that $G \leq C, F$. Since $C$ is minimal, it follows that $G=C$, so $C \leq F$, whence $C$ is the least element of $\mathcal{F}$.

For (2) implies (3), let $C_{1} \geq C_{2} \geq C_{3} \geq \ldots$ be a descending chain. Then $\mathcal{F}=\left\{C_{i}\right\}_{i \in \mathbb{N}}$ is clearly a directed subset, so it has a least element, say $C_{n}$. So we must have $C_{k}=C_{n}$ for all $k>n$, hence $\mathcal{C}$ satisfies (3).

Finally, we show by contraposition that (3) follows from (1). So assume that $\mathcal{C}$ does not satisfy the descending chain condition. Hence we can construct a sequence $C_{1} \geq C_{2} \geq \ldots$ that does not terminate. The set $\mathcal{F}=\left\{C_{n}: n \in \mathbb{N}\right\}$ is then a non-empty subset of $\mathcal{C}$ without a minimal element. Thus $\mathcal{C}$ does not satisfy (1).

There exists also a notion dual to the notion of an Artinian poset.

Definition 5 Let $\mathcal{C}$ be a poset. Then $\mathcal{C}$ is called Noetherian if is satisfies one of the following equivalent conditions:

1) Every non-empty subset contains a maximal element;

2) All non-empty directed subsets of $\mathcal{C}$ have a greatest element;

3) $\mathcal{C}$ satisfies the ascending chain condition: if we have a sequence of elements $C_{1} \leq$ $C_{2} \leq \ldots$ in $\mathcal{C}$, i.e., a countable ascending chain, then the sequence stabilizes, i.e., there is an $n \in \mathbb{N}$ such that $C_{k}=C_{n}$ for all $k>n$.

The following proposition can be found in [26] as Theorem 4.21.

Proposition 3 (Principle of Artinian induction) Let $\mathcal{C}$ be an Artinian poset and $\mathcal{P}$ a property such that:

1. $\mathcal{P}(C)$ is true for each minimal $C \in \mathcal{C}$ (induction basis);

2. $\mathcal{P}(B)$ is true for all $B<C$ implies that $\mathcal{P}(C)$ is true (induction step).

Then $\mathcal{P}(C)$ is true for each $C \in \mathcal{C}$.

Proof Assume that $\mathcal{F}=\{C \in \mathcal{C}: \mathcal{P}(C)$ is not true $\}$ is non-empty. Since $\mathcal{C}$ is Artinian, this means that $\mathcal{F}$ has a minimal element $C$. Hence $\mathcal{P}(B)$ is true for all elements $B<C$, so $\mathcal{P}(C)$ is true by the induction step, contradicting the definition of $\mathcal{F}$. 
Definition 6 Let $\mathcal{C}$ be a poset. Then $\mathcal{C}$ is called graded if one can define a function $d: \mathcal{C} \rightarrow$ $\mathbb{N}$, called a rank function such that:

(i) $d(C)=1$ for each $C \in \min \mathcal{C}$;

(ii) $d\left(C_{1}\right)<d\left(C_{2}\right)$ for each $C_{1}, C_{2} \in \mathcal{C}$ such that $C_{1}<C_{2}$;

(iii) $C_{2}$ is a cover of $C_{1}$ if and only if $C_{1} \leq C_{2}$ and $d\left(C_{2}\right)=d\left(C_{1}\right)+1$ for each $C_{1}, C_{2} \in \mathcal{C}$.

There is no standard definition of a graded poset. For instance, in [36] condition (i) is dropped and $\mathbb{Z}$ is taken as codomain of rank functions. On the other hand, [30] assumes condition (i), but not condition (ii). For our purposes, it is convenient to combine both definitions. The next three lemmas are now easy to prove.

Lemma 6 Let $\mathcal{C}$ be a graded poset with rank funcion $d: \mathcal{C} \rightarrow \mathbb{N}$. Then $\mathcal{C}$ is Artinian. If the range of $d$ is bounded from above, $\mathcal{C}$ is Noetherian as well.

Proof Let $\mathcal{F} \subseteq \mathcal{C}$ a non-empty filtered subset. Then $d[\mathcal{F}] \subseteq \mathbb{N}$ is non-empty, so it contains a least element $n$. Let $F_{1}, F_{2} \in \mathcal{F}$ such that $d\left(F_{1}\right)=d\left(F_{2}\right)=n$. Since $\mathcal{F}$ is filtered, there is an $F \in \mathcal{F}$ such that $F \leq F_{1}, F_{2}$. Assume that $F \neq F_{1}$. Then $F<F_{1}$, so $d(F)<d\left(F_{1}\right)=n$ contradicting the minimality of $n$. Hence we must have $F=F_{1}$ and in a similar way, we find that $F=F_{2}$. So there is a unique element $F \in \mathcal{F}$ such that $d(F)=n$. Let $F^{\prime} \in \mathcal{F}$. Since $\mathcal{F}$ is filtered, there is some $F^{\prime \prime} \in \mathcal{F}$ such that $F^{\prime \prime} \leq F, F^{\prime}$. Again if $F^{\prime \prime}<F$, we find $d\left(F^{\prime \prime}\right)<n$ contradicting the minimality of $n$, so $F^{\prime \prime}=F$. It follows that $F \leq F^{\prime}$, so $F$ is the least element of $\mathcal{F}$. We conclude that $\mathcal{C}$ is Artinian. Now assume that $d[\mathcal{C}] \subseteq \mathbb{N}$ has an upper bound, then the proof that $\mathcal{C}$ is Noetherian follows in an analogous way.

Lemma 7 Let $\mathcal{C}$ be a graded poset. Then its rank function $d: \mathcal{C} \rightarrow \mathbb{N}$ is unique.

Proof By Lemma 6, $\mathcal{C}$ is Artinian. Hence every non-empty subset has a minimal element, and in particular $\min \mathcal{C} \neq \varnothing$. Assume that $g: \mathcal{C} \rightarrow \mathbb{N}$ is a rank function. By definition of a rank function we have $g(M)=d(M)=1$ for each $M \in \min \mathcal{C}$.

Let $C \in \mathcal{C}$ such that $C \notin \min \mathcal{C}$. Assume that $d(B)=g(B)$ for each $B<C$. The set $\{d(B): B<C\} \subseteq \mathbb{N}$ is non-empty and bounded by $d(C)$, hence it must have a maximum $n$. As a consequence, there is some $B<C$ such that $d(B)=n$. Assume that $n+1 \neq d(C)$. Then $C$ does not cover $B$, hence there is some $B^{\prime} \in \mathcal{C}$ such that $B<B^{\prime}<C$. Thus $n=d(B)<d\left(B^{\prime}\right)$ contradicting the maximality of $n$. We conclude that $d(B)+1=d(C)$, so $C$ covers some $B$. Hence we obtain

$$
d(C)=d(B)+1=g(B)+1=g(C),
$$

so $d=g$ by Artinian induction.

Lemma 8 Let $\phi: \mathcal{C} \rightarrow \mathcal{D}$ be an order isomorphism between graded posets $\mathcal{C}$ and $\mathcal{D}$ with rank functions $d_{\mathcal{C}}$ and $d_{\mathcal{D}}$, respectively. Then $d_{\mathcal{C}}=d_{\mathcal{D}} \circ \phi$.

Proof Let $d=d_{\mathcal{D}} \circ \phi$. We check that $d$ is a rank function on $\mathcal{C}$. Let $C \in \min \mathcal{C}$, then $\phi(C) \in \min \mathcal{D}$, so $d(C)=d_{\mathcal{D}} \circ \phi(C)=1$. 
Let $C_{1}, C_{2} \in \mathcal{C}$ such that $C_{1}<C_{2}$. Then $\phi\left(C_{1}\right) \leq \phi\left(C_{2}\right)$, and since $\phi$ is injective, we cannot have equality. Hence $\phi\left(C_{1}\right)<\phi\left(C_{2}\right)$, so

$$
d\left(C_{1}\right)=d_{\mathcal{D}} \circ \phi\left(C_{1}\right)<d_{\mathcal{D}} \circ \phi\left(C_{2}\right)=d\left(C_{2}\right) .
$$

Finally, let $C_{1}, C_{2} \in \mathcal{C}$. Clearly, $C_{2}$ covers $C_{1}$ if and only if $\phi\left(C_{2}\right)$ covers $\phi\left(C_{1}\right)$ if and only if $d_{2}\left(\phi\left(C_{2}\right)\right)=d_{2}\left(\phi\left(C_{1}\right)\right)+1$ and $C_{1} \leq C_{2}$ if and only if $d\left(C_{2}\right)=d\left(C_{1}\right)+1$ and $C_{1} \leq C_{2}$.

Thus $d$ is a rank function on $\mathcal{C}$ and by Lemma7, we find $d_{1}=d$.

Lemma 9 Let $A$ be a finite-dimensional $C^{*}$-algebra. Then $\mathcal{C}(A)$ is graded with a rank function $\operatorname{dim}: \mathcal{C}(A) \rightarrow \mathbb{N}$ assigning to each element $C \in \mathcal{C}(A)$ its dimension. Moreover, the range of $\operatorname{dim}$ is bounded from above.

\section{Proof Trivial.}

Combining Lemmas 6 and 9, we find that if $A$ is finite dimensional, then $\mathcal{C}(A)$ is both Artinian and Noetherian. We shall prove that the converse holds as well.

Lemma 10 Let $A$ be a $C^{*}$-algebra. Then every element of $\mathcal{C}(A)$ is contained in a maximal element of $\mathcal{C}(A)$. In particular, the set $\max \mathcal{C}(A)$ is non-empty.

Proof Let $C \in \mathcal{C}(A)$ and let $\mathcal{S}=\{D \in \mathcal{C}(A): C \subseteq D\}$. Then $\mathcal{S}$ is non-empty, so if $\mathcal{C}(A)$ is Noetherian, we immediately find that $\mathcal{S}$ contains a maximal element. If $\mathcal{C}(A)$ is not Noetherian, we need Zorn's Lemma. Let $\mathcal{D}=\left\{D_{i}\right\}_{i \in I}$ be a chain in $\mathcal{S}$. Then $\mathcal{D}$ is certainly directed, hence it must have a supremum $\bigvee \mathcal{D}$ by Proposition 1 . Since $\bigvee \mathcal{D}$ clearly contains $C$, we have $\bigvee \mathcal{D} \in \mathcal{S}$. So for every chain $\mathcal{C}(A)$, there is an upper bound for the chain in $\mathcal{S}$. By Zorn's Lemma it follows that $\mathcal{S}$ contains a maximal element $M$. Now, $M$ must also be maximal in $\mathcal{C}(A)$, since if there is some $A \in \mathcal{C}(A)$ such that $M \subseteq A$, then $C \subseteq A$, so $A \in \mathcal{S}$. By the maximality of $M$ with respect to $\mathcal{S}$, it follows that $A$ must be equal to $M$.

Proposition 4 Let $A$ be a $C^{*}$-algebra and $M$ a maximal commutative *-subalgebra. If $M$ is finite dimensional, then A must be finite dimensional as well.

This statement can be found in [27] as Exercise 4.12. The solution of this exercise can be found in [28].

In particular, when $A=\mathrm{M}_{n}(\mathbb{C})$, there is a nice characterization of the maximal commutative $\mathrm{C}^{*}$-subalgebras of $\mathcal{C}(A)$.

Lemma 11 Let $A=\mathrm{M}_{n}(\mathbb{C})$ and $M \in \max \mathcal{C}(A)$. Then $M$ is $n$-dimensional and there is some $u \in \mathrm{SU}(n)$ such that $M=\left\{u d u^{*}: d \in D_{n}\right\}$, where $D_{n}$ is the commutative $C^{*}$-subalgebra of A consisting of all diagonal matrices.

Proof See [21, Example 5.3.5].

Definition 7 Let $X$ be a topological space with topology $\mathcal{O}(X)$. Then $X$ is called Noetherian if the poset $\mathcal{O}(X)$ ordered by inclusion is Noetherian. 
Lemma 12 [19, Exercise I.1.7] Let $X$ be a topological space. If $X$ is Noetherian and Hausdorff, then $X$ must be finite.

Proof First we show that every subset of $X$ is compact. So if $Y \subseteq X$, let $\mathcal{U}$ be a cover of $Y$. Let $\mathcal{V}$ be the set of all finite unions of elements of $\mathcal{U}$. Then $\mathcal{V}$ covers $Y$ as well, and moreover, $\mathcal{V}$ is directed. Since $\mathcal{O}(X)$ is Noetherian, $\mathcal{V}$ has a greatest element $V$. Now, $V$ contains every element of $\mathcal{V}$ and since $\mathcal{V}$ covers $Y$, we find that $V$ must contain $Y$. It follows from the definition of $\mathcal{V}$ that $V$ can be written as a finite union of elements of $\mathcal{U}$, so $\mathcal{U}$ has a finite subcover. Thus $Y$ is compact. Now let $x \in X$. Then $X \backslash\{x\}$ is compact, hence closed. Hence $\{x\}$ is open, and it follows that $X$ is discrete. Since $X$ itself is compact, $X$ must be finite.

Proposition 5 Let $A$ be a $C^{*}$-algebra. Then $A$ is finite dimensional if and only if $\mathcal{C}(A)$ is Artinian if and only if $\mathcal{C}(A)$ is Noetherian.

Proof Assume that $A$ is finite dimensional. By Lemma 9, $\mathcal{C}(A)$ has a rank function whose range is bounded from above. By Lemma $6, \mathcal{C}(A)$ is both Artinian and Noetherian.

Assume that $A$ is not finite dimensional. By Lemma 10, $\mathcal{C}(A)$ has a maximal element $M$. By Proposition 4, it follows that $M$ cannot be finite dimensional. Since $M$ is a unital commutative $\mathrm{C}^{*}$-algebra, the Gel'fand-Naimark Theorem assures that $M=C(X)$ for some compact Hausdorff space $X$, which must have an infinite number of points since $A$ is infinite dimensional.

We construct a descending chain in $\mathcal{C}(A)$ as follows. First choose a countable subset $\left\{x_{1}, x_{2}, x_{3}, \ldots\right\}$ of $X$. Let

$$
C_{n}=\left\{f \in C(X): f\left(x_{1}\right)=\ldots=f\left(x_{n}\right)\right\}
$$

for each $n \in \mathbb{N}$. Clearly, we have $C_{1} \supseteq C_{2} \supseteq C_{3} \supseteq \ldots$.. Assume that $i<j$. Then $\left\{x_{1}, \ldots, x_{i}\right\}$ and $\left\{x_{j}\right\}$ are disjoint closed sets, hence Urysohn's Lemma assures the existence of some $f \in C(X)$ such that $f\left[\left\{x_{1}, \ldots, x_{i}\right\}\right]=\{1\}$ and $f\left(x_{j}\right)=0$. Clearly, $f \in C_{i}$, but $f \notin C_{j}$. This shows that $C_{i} \neq C_{j}$, so the chain is descending, but it never stabilizes.

We construct an ascending chain in $\mathcal{C}(A)$ as follows. First we notice that since $X$ is infinite and Hausdorff, Lemma 12 implies that $X$ is not Noetherian. So there is an ascending chain $O_{1} \subseteq O_{2} \subseteq \ldots$ of open subsets of $X$ that does not stabilize. For each $i \in \mathbb{N}$, let $F_{i}=X \backslash O_{i}$. Then $F_{1} \supseteq F_{2} \supseteq \ldots$ is a descending chain of closed subsets of $X$, which does not stabilize. For each $i \in I$ let $C_{i}=C_{F_{i}}$. Then $C_{i}$ is a $C^{*}$-subalgebra of $C(X)$ and if $i \leq j$, we have $F_{i} \supseteq F_{j}$, so $C_{i} \subseteq C_{j}$. Moreover, if $i<j$ and $F_{i} \neq F_{j}$, then there is some $x \in F_{i}$ such that $x \notin F_{j}$. By Urysohn's Lemma, there is an $f \in C(X)$ such that $f(x)=0$ and $f(y)=1$ for each $y \in F_{j}$. Hence $f \in C_{j}$, but $f \notin C_{i}$. It follows that $C_{i} \neq C_{j}$, so $C_{1} \subseteq C_{2} \subseteq \ldots$ is an ascending chain that does not stabilize.

Thus $\mathcal{C}(A)$ contains an ascending chain as well as a descending chain, neither of which stabilizes. Hence $\mathcal{C}(A)$ can be neither Noetherian nor Artinian.

Recall that the center of a $C^{*}$-algebra $A$ is the set $\{x \in A: x y=y x \forall y \in A\}$, which is usually denoted by $Z(A)$.

Lemma 13 Let $A$ be a $C^{*}$-algebra. Then $Z(A)$ is a commutative $C^{*}$-subalgebra. Moreover, $Z(A)$ is the intersection of all maximal commutative $C^{*}$-subalgebras of $A$. 
Proof For each $y \in A$, consider the map $f_{y}: A \rightarrow A$ given by $x \mapsto x y-y x$. Clearly this is continuous and linear, so $\operatorname{ker} f_{y}$ is a closed linear subspace of $A$. Hence $Z(A)=$ $\bigcap_{y \in A} \operatorname{ker} f_{y}$ is a closed linear subspace as well. If $x, y \in Z(A)$ and $z \in A$, then $x y z=$ $x z y=z x y$, so $x y \in Z(A)$. Moreover, $x^{*} z=\left(z^{*} x\right)^{*}=\left(x z^{*}\right)^{*}=z x^{*}$, so $x^{*} \in Z(A)$. Clearly $x y=y x$, and $1_{A} \in Z(A)$, hence $Z(A) \in \mathcal{C}(A)$.

Let $x \in \bigcap \max \mathcal{C}(A)$, i.e., $x \in M$ for each maximal $M \in \mathcal{C}(A)$. Let $y \in A$. Then $y$ can be written as a linear combination of two self-adjoint elements $a_{1}, a_{2}$. If $a \in A$ is self-adjoint, then $C^{*}(a, 1)$ is a commutative $C^{*}$-subalgebra of $A$ containing $a$. By Lemma 10 , it follows that there are $M_{1}, M_{2} \in \max \mathcal{C}(A)$ such that $a_{i} \in M_{i}$ for $i=1$, 2. Since $x \in M_{1}, M_{2}$, it follows that $x$ commutes with both $a_{1}$ and $a_{2}$. Hence $x$ commutes with $y$, so $x \in Z(A)$. Thus $\bigcap \max \mathcal{C}(A) \subseteq Z(A)$.

Now assume that $x \in Z(A)$. Since $x$ commutes with all elements of $A$, it commutes in particular with $x^{*}$. Hence $x$ is normal. We have $x^{*} \in Z(A)$ as well, for $Z(A)$ is a *-subalgebra of $A$. Let $M \in \max \mathcal{C}(A)$. Then $M \cup\left\{x, x^{*}\right\}$ is a set of mutually commuting elements, which is *-closed and contains $1_{A}$. It follows that $C^{*}\left(M \cup\left\{x, x^{*}\right\}\right)$, the $\mathrm{C}^{*}$-subalgebra of $A$ generated by $M \cup\left\{x, x^{*}\right\}$, is commutative. Since $M$ is maximal, $C^{*}\left(M \cup\left\{x, x^{*}\right\}\right)$ must be equal to $M$. As a consequence, $x \in M$, so we find that $x$ is contained in every maximal commutative $C^{*}$-subalgebra of $A$. Hence $Z(A) \subseteq \bigcap \max \mathcal{C}(A)$.

Lemma 14 Let $A_{1}, \ldots, A_{n}$ be $C^{*}$-algebras. Then

$$
Z\left(\bigoplus_{i=1}^{n} A_{i}\right)=\bigoplus_{i=1}^{n} Z\left(A_{i}\right)
$$

Proof This follows directly from the fact that multiplication on $\bigoplus_{i=1}^{n} A_{i}$ is calcultated coordinatewisely.

Lemma 15 Let $A_{1}, \ldots, A_{n}$ be $C^{*}$-algebras. Let $A=\bigoplus_{i=1}^{n} A_{i}$ and $C \in \mathcal{C}(A)$ such that $Z(A) \subseteq C$. Then there are $C_{i} \in \mathcal{C}\left(A_{i}\right)$ such that $Z\left(A_{i}\right) \subseteq C_{i}$ and $C=\bigoplus_{i=1}^{n} C_{i}$.

Proof Let $p_{i}: A \rightarrow A_{i}$ be the projection on the $i$-th factor. Then we obtain an order morphism $\mathcal{C}\left(p_{i}\right): \mathcal{C}(A) \rightarrow \mathcal{C}\left(A_{i}\right)$. Let $C_{i}=\mathcal{C}\left(p_{i}\right)(C)$, or equivalently, $C_{i}=p_{i}[C]$. Then

$$
Z\left(A_{i}\right)=p_{i}\left[\bigoplus_{i=1}^{n} Z\left(A_{i}\right)\right]=p_{i}[Z(A)] \subseteq p_{i}[C]=C_{i}
$$

for each $i \in I$.

Let $c \in C$, then $p_{i}(c) \in C_{i}$ for each $i=1, \ldots, n$, so $c=p_{1}(c) \oplus \ldots \oplus p_{n}(c)$. Thus $c \in \bigoplus_{i=1}^{n} C_{i}$, hence $C \subseteq \bigoplus_{i=1}^{n} C_{i}$.

Let $c_{1} \oplus \ldots \oplus c_{n} \in \bigoplus_{i=1}^{n} C_{i}$. This means that for each $i=1, \ldots, n$ there is a $d^{i} \in C$ such that $p_{i}\left(d^{i}\right)=c_{i}$. Here $d^{i}=d_{1}^{i} \oplus \ldots \oplus d_{n}^{i}$, with $d_{j}^{i} \in A_{j}$, and in particular we have $d_{i}^{i}=c_{i}$. For each $j=1, \ldots, n$, let $e^{j} \in A$ be the element $e_{1}^{j} \oplus \ldots \oplus e_{n}^{j}$, with

$$
e_{i}^{j}= \begin{cases}1_{A_{i}} & i=j \\ 0_{A_{i}} & i \neq j\end{cases}
$$


Here $1_{A_{i}}$ and $0_{A_{i}}$ denote the unit and the zero of $A_{i}$, respectively. Since $1_{A_{i}}$ and $0_{A_{i}}$ are elements of $Z\left(A_{i}\right)$, Lemma 14 assures that $e^{j} \in Z(A)$. Since $Z(A) \subseteq C$, we find that $e^{j} \in C$. It follows that $f^{j}=e^{j} d^{j} \in C$. Here $f^{j}=f_{1}^{j} \oplus \ldots \oplus f_{n}^{j}$ with

$$
f_{i}^{j}= \begin{cases}c_{i} & i=j \\ 0_{A_{i}} & i \neq j\end{cases}
$$

Now,

$$
f^{1}+\ldots+f^{n}=c_{1} \oplus \ldots \oplus c_{n}=c
$$

and since $f^{j} \in C$, it follows that $c \in C$. So $C=\bigoplus_{i=1}^{n} C_{i}$.

Proposition 6 Let $A_{1}, \ldots, A_{n}$ be $C^{*}$-algebras and let $A=\bigoplus_{i=1}^{n} A_{i}$. Define the map $\iota: \prod_{i=1}^{n} \mathcal{C}\left(A_{i}\right) \rightarrow \mathcal{C}(A)$ by $\left\langle C_{1}, \ldots, C_{n}\right\rangle \mapsto C_{1} \oplus \ldots \oplus C_{n}$, and the map $\pi: \mathcal{C}(A) \mapsto$ $\prod_{i=1}^{n} \mathcal{C}\left(A_{i}\right)$ by $\mathcal{C}\left(p_{1}\right) \times \ldots \times \mathcal{C}\left(p_{n}\right)$, where $p_{i}: A \rightarrow A_{i}$ denotes the projection on the $i$-th factor. Thus $\pi$ maps $C \in \mathcal{C}(A)$ to $\left\langle p_{1}[C], \ldots, p_{n}[C]\right\rangle$. Then:

(i) $\iota$ is an embedding of posets;

(ii) $\pi$ is surjective;

(iii) $\pi \circ \iota=1_{\prod_{i=1}^{n} \mathcal{C}\left(A_{i}\right)}$ and $1_{\mathcal{C}(A)} \leq \iota \circ \pi$;

(iv) the restriction of $\iota$ to a map $\prod_{i=1}^{n} \mathcal{C}\left(A_{i}\right) \rightarrow \uparrow Z(A)$ is an order isomorphism with inverse $\pi$.

Proof For each $i=1, \ldots, n$, let $C_{i} \in \mathcal{C}\left(A_{i}\right)$. Then $C_{1} \oplus \ldots \oplus C_{n}$ is clearly a commutative $\mathrm{C}^{*}$-algebra of $A$, which is unital since $1_{A}=1_{A_{1}} \oplus \ldots \oplus 1_{A_{n}}$. Hence the image of $\iota$ lies in $\mathcal{C}(A)$, so $\iota$ is well defined. Furthermore, we remark that $\mathcal{C}\left(p_{i}\right): \mathcal{C}(A) \rightarrow \mathcal{C}\left(A_{i}\right)$ is an order morphism by Lemma 3.

(i) Let $\left\langle C_{1}, \ldots, C_{n}\right\rangle$ and $\left\langle D_{1}, \ldots, D_{n}\right\rangle$ be elements of $\prod_{i=1}^{n} \mathcal{C}\left(A_{i}\right)$. Then

$$
\left\langle C_{1}, \ldots, C_{n}\right\rangle \leq\left\langle D_{1}, \ldots, D_{n}\right\rangle
$$

implies $C_{i} \subseteq D_{i}$ for each $i=1, \ldots, n$. Hence $C_{1} \oplus \ldots \oplus C_{n} \subseteq D_{1} \oplus \ldots \oplus D_{n}$, which says exactly that

$$
\iota\left(\left\langle C_{1}, \ldots, C_{n}\right\rangle\right) \subseteq \iota\left(\left\langle D_{1}, \ldots, D_{n}\right\rangle\right) .
$$

Conversely, if $\iota\left(\left\langle C_{1}, \ldots, C_{n}\right\rangle\right) \subseteq \iota\left(\left\langle D_{1}, \ldots, D_{n}\right\rangle\right)$, we have

$$
C_{1} \oplus \ldots \oplus C_{n} \subseteq D_{1} \oplus \ldots \oplus D_{n} .
$$

If we let act $\mathcal{C}\left(p_{i}\right)$ on both sides of this inclusion, we obtain $C_{i} \subseteq D_{i}$ for each $i=$ $1, \ldots, n$. Hence $\left\langle C_{1}, \ldots, C_{n}\right\rangle \leq\left\langle D_{1}, \ldots, D_{n}\right\rangle$. Thus $\iota$ is an embedding of posets.

(ii) Let $\left\langle C_{1}, \ldots, C_{n}\right\rangle \in \prod_{i=1}^{n} \mathcal{C}\left(A_{i}\right)$. If $C=C_{1} \oplus \ldots \oplus C_{n}$, then $C \in \mathcal{C}(A)$ and

$$
\pi(C)=\left\langle\mathcal{C}\left(p_{1}\right)(C), \ldots, \mathcal{C}\left(p_{n}\right)(C)\right\rangle=\left\langle p_{1}[C], \ldots, p_{n}[C]\right\rangle=\left\langle C_{1}, \ldots, C_{n}\right\rangle .
$$

(iii) Let $\left\langle C_{1}, \ldots, C_{n}\right\rangle \in \prod_{i=1}^{n} \mathcal{C}\left(A_{i}\right)$. Let $C=\iota\left(\left\langle C_{1}, \ldots, C_{n}\right\rangle\right)$. Then

$$
C=C_{1} \oplus \ldots \oplus C_{n},
$$

and by the calculation in (ii), we obtain $\pi(C)=\left\langle C_{1}, \ldots, C_{n}\right\rangle$. It follows that $\pi \circ \iota=$ $1_{\prod_{i=1}^{n} \mathcal{C}\left(A_{i}\right)}$ holds.

Let $C \in \mathcal{C}(A)$. Then

$$
\iota \circ \pi(C)=\iota\left(\left\langle p_{1}[C], \ldots, p_{n}[C]\right\rangle\right)=p_{1}[C] \oplus \ldots \oplus p_{n}[C] .
$$


Let $c \in C$. Since $C \subseteq A$, and $A=\bigoplus_{i=1}^{n} A_{i}$, there are $c_{i} \in A_{i}$ for each $i=$ $1, \ldots, n$ such that $c=c_{1} \oplus \ldots \oplus c_{n}$. Hence $c_{i}=p_{i}(c)$, and we find

$$
c=p_{1}(c) \oplus \ldots \oplus p_{n}(c),
$$

so $c \in p_{1}[C] \oplus \ldots \oplus p_{n}[C]$. But

$$
p_{1}[C] \oplus \ldots \oplus p_{n}[C]=\iota\left(\left\langle p_{1}[C], \ldots, p_{n}[C]\right\rangle\right)=\iota \circ \pi(C) .
$$

Hence $c \in \iota \circ \pi(C)$, so $C \subseteq \iota \circ \pi(C)$. We conclude that $1_{\mathcal{C}(A)} \leq \iota \circ \pi$.

(iv) In order to show that $\iota$ restricts to an order isomorphism

$$
\prod_{i=1}^{n} \uparrow Z\left(A_{i}\right) \rightarrow \uparrow Z(A)
$$

with inverse $\pi$, it is enough to show that $\iota \circ \pi(C)=C$ for each $C \in \uparrow Z(A)$. Then the statement follows directly from the equality in (iii). So let $C \in \mathcal{C}(A)$ such that $Z(A) \subseteq C$. Then Lemma 15 assures that there are $C_{i} \in \mathcal{C}\left(A_{i}\right)$ for each $i=1, \ldots, n$ such that $C=C_{1} \oplus \ldots \oplus C_{n}$ and $Z\left(A_{i}\right) \subseteq C_{i}$ for each $i=1, \ldots, n$. Then $p_{i}[C]=C_{i}$, hence

$$
\iota \circ \pi(C)=\iota\left(\left\langle p_{1}[C], \ldots, p_{n}[C]\right\rangle\right)=\iota\left(\left\langle C_{1}, \ldots, C_{n}\right\rangle\right)=C_{1} \oplus \ldots \oplus C_{n}=C .
$$

Proposition 7 Let $A=\bigoplus_{i=1}^{k} \mathrm{M}_{n_{i}}(\mathbb{C})$, where $k, n_{1}, \ldots, n_{k} \in \mathbb{N}$. Then

$$
[Z(A), M] \cong \prod_{i=1}^{k} \mathcal{C}\left(\mathbb{C}^{n_{i}}\right)
$$

for each $M \in \max \mathcal{C}(A)$.

Proof By Proposition 6, there is an order morphism $\pi: \mathcal{C}(A) \rightarrow \prod_{i=1}^{j} \mathcal{C}\left(\mathrm{M}_{n_{i}}(\mathbb{C})\right)$ whose restriction to $\uparrow Z(A)$ is an order isomorphism with inverse $\iota$.

Let $M \in \max \mathcal{C}(A)$. By Lemma 13 we have $Z(A) \subseteq M$, so $M \in \uparrow Z(A)$, hence $M \in \max \uparrow Z(A)$. Since $\pi$ is an order isomorphism, $\pi(M)$ is a maximal element of $\prod_{i=1}^{k} \mathcal{C}\left(\mathrm{M}_{n_{i}}(\mathbb{C})\right)$. Clearly there are $M_{n_{i}} \in \max \mathcal{C}\left(\mathrm{M}_{n_{i}}(\mathbb{C})\right)$ for each $i=1, \ldots, k$ such that $\pi(M)=\left\langle M_{n_{1}}, \ldots, M_{n_{k}}\right\rangle$. It follows that $\downarrow \pi(M)=\downarrow M_{n_{1}} \times \ldots \times \downarrow M_{n_{k}}$.

Since $\iota$ is the inverse of $\pi$ and has codomain $\uparrow Z(A)$, we find that

$$
\iota[\downarrow \pi(M)]=\downarrow \iota \circ \pi(M) \cap \uparrow Z(A)=\downarrow M \cap \uparrow Z(A)=[Z(A), M] .
$$

Hence the restriction $\iota: \downarrow M_{n_{1}} \times \ldots \times \downarrow M_{n_{k}} \rightarrow[Z(A), M]$ is an order isomorphism.

Notice that all maximal elements of $\mathcal{C}\left(\mathrm{M}_{n_{i}}(\mathbb{C})\right)$ are *-isomorphic by Lemma 11 . More specificaly, $M_{n_{i}} \in \max \mathcal{C}\left(\mathrm{M}_{m_{i}}(\mathbb{C})\right)$ is *-isomorphic to $D_{n_{i}}$. Since

$$
D_{n_{i}}=\left\{\operatorname{diag}\left(\lambda_{1}, \ldots, \lambda_{n_{i}}\right): \lambda_{1}, \ldots, \lambda_{n_{i}} \in \mathbb{C}\right\},
$$

we find that $M_{n_{i}}$ is *-isomorphic to $\mathbb{C}^{n_{i}}$. Hence we can find an injective *-homomorphism $f: \mathbb{C}^{n_{i}} \rightarrow \mathrm{M}_{n_{i}}(\mathbb{C})$ such that $f\left[C^{n_{i}}\right]=M_{n_{i}}$. By Proposition 2, we find that $\mathcal{C}(f)$ : $\mathcal{C}\left(\mathbb{C}^{n_{i}}\right) \rightarrow \mathcal{C}\left(\mathrm{M}_{n_{i}}(\mathbb{C})\right)$ is an order embedding with image $\downarrow M_{n_{i}}$. We conclude that $\mathcal{C}\left(\mathbb{C}^{n_{i}}\right) \cong$ $\downarrow M_{n_{i}}$ in $\mathcal{C}\left(\mathrm{M}_{n_{i}}(\mathbb{C})\right)$. Hence $[Z(A), M] \cong \prod_{i=1}^{k} \mathcal{C}\left(\mathbb{C}^{n_{i}}\right)$. 
Definition 8 [4, III.8] Let $\mathcal{C}$ be a lattice. Then $\mathcal{C}$ is called directly indecomposable if $\mathcal{C} \cong$ $\mathcal{C}_{1} \times \mathcal{C}_{2}$ for some lattices $\mathcal{C}_{1}, \mathcal{C}_{2}$ implies that either $\mathcal{C}_{1}=\mathbf{1}$ and $\mathcal{C}_{2}=\mathcal{C}$ or $\mathcal{C}_{1}=\mathcal{C}$ and $\mathcal{C}_{2}=\mathbf{1}$, where $\mathbf{1}$ denotes the one-point poset.

The following result is also known as Hashimoto's Theorem.

Theorem 3 Let $\mathcal{C}$ be a lattice with a least element 0 . If there are two direct decompositions of $\mathcal{C}$

$$
\begin{aligned}
\mathcal{C} & =\mathcal{A}_{1} \times \ldots \times \mathcal{A}_{n} ; \\
\mathcal{C} & =\mathcal{B}_{1} \times \ldots \times \mathcal{B}_{m},
\end{aligned}
$$

where $n, m \in \mathbb{N}$, then there are lattices $\mathcal{C}_{i j}, i=1, \ldots, n$ and $j=1, \ldots, m$ such that

$$
\begin{aligned}
& \mathcal{A}_{i}=\mathcal{C}_{i 1} \times \ldots \times \mathcal{C}_{i m} ; \\
& \mathcal{B}_{j}=\mathcal{C}_{1 j} \times \ldots \times \mathcal{C}_{n j} .
\end{aligned}
$$

Proof [16, Theorem III.4.2]

Corollary 1 Let $\mathcal{A}_{1} \times \ldots \times \mathcal{A}_{n}=\mathcal{B}_{1} \times \ldots \times \mathcal{B}_{m}$, where $n, m \in \mathbb{N}$ and the $\mathcal{A}_{i}$ and $\mathcal{B}_{i}$ are directly indecomposable lattices. Then $n=m$, and there is some permutation $\pi$ : $\{1, \ldots, n\} \rightarrow\{1, \ldots, n\}$ such that $A_{i} \cong B_{\pi(i)}$ for each $i=1, \ldots, n$.

Definition 9 Let $\mathcal{C}$ be a bounded lattice and $C \in \mathcal{C}$. Then $D \in \mathcal{C}$ is called a complement of $C$ if $C \wedge D=0$ and $C \vee D=1$.

The next Proposition is actually an application of [16, Theorem III.4.1].

Proposition 8 Let $\mathcal{C}$ be a bounded lattice. If 0 and 1 are the only elements of $\mathcal{C}$ with a unique complement (namely each other), then $\mathcal{C}$ is directly indecomposable.

Proof Let $D \in \mathcal{C}$ be a complement of 1 . Then $D=1 \wedge D=0$. If $D$ is a complement of 0 , then $D=0 \vee D=1$. Thus 0 and 1 are each other's unique complement.

Now assume that there exists an order isomorphism $\phi: \mathcal{C}_{1} \times \mathcal{C}_{2} \rightarrow \mathcal{C}$, where $\mathcal{C}_{1}$ and $\mathcal{C}_{2}$ are bounded lattices not equal to 1 . This last condition implies that $\langle 1,1\rangle \neq\langle 1,0\rangle \neq\langle 0,0\rangle$. Let $C=\phi(\langle 1,0\rangle)$. Then it follows that $1 \neq C \neq 0$. Clearly $\langle 0,1\rangle$ is a complement of $\langle 1,0\rangle$ in $\mathcal{C}_{1} \times \mathcal{C}_{2}$, but it is also unique. Let $\left\langle D_{1}, D_{2}\right\rangle$ be a complement of $\langle 0,1\rangle$. Since suprema and infima are calculated componentwise, we find

$$
\begin{aligned}
& \left\langle D_{1}, 0\right\rangle=\left\langle D_{1} \wedge 1, D_{2} \wedge 0\right\rangle=\left\langle D_{1}, D_{2}\right\rangle \wedge\langle 1,0\rangle=\langle 0,0\rangle ; \\
& \left\langle 1, D_{2}\right\rangle=\left\langle D_{1} \vee 1, D_{2} \vee 0\right\rangle=\left\langle D_{1}, D_{2}\right\rangle \vee\langle 1,0\rangle=\langle 1,1\rangle,
\end{aligned}
$$

hence $D_{1}=0, D_{2}=1$. Thus $\left\langle D_{1}, D_{2}\right\rangle=\langle 0,1\rangle$, whence $\langle 1,0\rangle$ indeed has a unique complement. Since $\phi$ is an order isomorphism, $\phi$ preserves suprema and infima, hence $C$ has a complement $D=\phi(\langle 0,1)\rangle$. Now assume that $C$ has another complement $D^{\prime}$. Since $\phi$ is an order isomorphism, it follows that $\phi^{-1}\left(D^{\prime}\right)$ is a complement of $\langle 1,0\rangle$, and by uniqueness of this complement, we obtain $\phi^{-1}\left(D^{\prime}\right)=\langle 0,1\rangle$. We find that

$$
D=\phi(\langle 0,1\rangle)=\phi \circ \phi^{-1}\left(D^{\prime}\right)=D^{\prime} .
$$

The statement follows now by contraposition. 
Proposition 9 Let $A$ be a commutative finite-dimensional $C^{*}$-algebra. Then $\mathcal{C}(A)$ is a directly indecomposable lattice.

Proof By Lemma 5, $\mathcal{C}(A)$ is a bounded lattice. Let $X$ be the spectrum of $A$.

If $X$ is a singleton set, then $A$ is one-dimensional, and $\mathcal{C}(A)=\left\{\mathbb{C} 1_{A}\right\}$, so $\mathcal{C}(A)=\mathbf{1}$, the one-point lattice, and there is nothing to prove. If $X$ is a two-point set, then $\mathcal{C}(A)=$ $\left\{A, \mathbb{C} 1_{A}\right\}$. So $\mathcal{C}(A)$ contains no other elements than a greatest and a least one, and is therefore certainly directly indecomposable.

Assume that $X$ has at least three points. Let $B \in \mathcal{C}(A)$, assumed not equal to $\mathbb{C} 1_{A}$ or $A$. By Lemma 2, we have $B=\bigcap_{x \in X} C_{[x]_{B}}$. Since $X$ is finite, it follows that $X / \sim_{B}$ is finite as well. Notice that we cannot have $[x]_{B}=\{x\}$ for all $x \in X$, otherwise $B=C(X)=A$. Neither can $X / \sim_{B}$ be a singleton set, since otherwise $B=\mathbb{C} 1_{A}$. For each element $[x]_{B}$ in $X / \sim_{B}$, choose a representative $x$. Let $K$ be the set of representatives. Notice that $K$ is not a singleton set, since $X / \sim_{B}$ contains at least two elements. Also notice that $K$ is not unique, since there is at least one $[x]_{B} \in X / \sim_{B}$ containing two or more points. Since $X$ is discrete, it follows that $K$ is closed.

Let $f \in B \cap C_{K}$ and let $x, y \in X$ be points such that $x \neq y$. If $[x]_{B}=[y]_{B}$, then $f(x)=f(y)$. If $[x]_{B} \neq[y]_{B}$, then there are $x^{\prime}, y^{\prime} \in K$ such that $x^{\prime} \in[x]_{B}$ and $y^{\prime} \in[y]_{B}$. Since $f \in C_{K}$, we find that $f\left(x^{\prime}\right)=f\left(y^{\prime}\right)$. Since $f \in B$, we obtain $f(x)=f\left(x^{\prime}\right)$ and $f(y)=f\left(y^{\prime}\right)$. Combining all equalities gives $f(x)=$ $f(y)$. So in all cases, $f(x)=f(y)$. So $f$ must be constant, and we conclude that $B \cap C_{K}=\mathbb{C} 1_{A}$.

Since $\mathcal{C}(A)$ is a lattice, $B \vee C_{K}$ exists. Let $f \in C(X)$. Let $g: X \rightarrow \mathbb{C}$ be defined by $g(x)=f(k)$ if $x \in[k]_{B}$, where $k \in K$. Notice that is well defined, since $K$ is a collection of representatives. Moreover, since $X$ is discrete, $g$ is continuous, so $g \in C(X)$. By definition, we have $g \in \bigcap_{x \in X} C_{[x]_{B}}$, so $g \in B$. Let $h=$ $f-g$. Then $h \in C(X)$, and if $k \in K$, we find $h(k)=f(k)-g(k)=0$, so $h$ is constant on $K$. We conclude that $f=g+h$ with $g \in B$ and $h \in C_{K}$. Hence $A=C(X)=B \vee C_{K}$.

We find that $C_{K}$ is a complement of $B$. However, $K$ is not unique, and therefore neither is $C_{K}$. We conclude that $A$ and $\mathbb{C} 1_{A}$ are the only elements with a unique complement, so $\mathcal{C}(A)$ is indirectly indecomposable.

The proof of this proposition is based on the proof of the directly indecomposability of partition lattices in [37]. More can be said about $\mathcal{C}(A)$ when $A$ is a commutative $\mathrm{C}^{*}$ algebra of dimension $n$, namely that $\mathcal{C}(A)$ is order isomorphic to the lattice of partitions of the set $\{1, \ldots, n\}$. We refer to [22] for a complete characterization of $\mathcal{C}(A)$ when $A$ is a commutative finite-dimensional $\mathrm{C}^{*}$-algebra.

We are now ready to prove the main result of this section.

Proof of Theorem 1 Let $A$ be a finite-dimensional $C^{*}$-algebra, and $B$ a $C^{*}$-algebra. Let $\phi: \mathcal{C}(A) \rightarrow \mathcal{C}(B)$ an order isomorphism. By Proposition $5, \mathcal{C}(A)$ is Noetherian, and so $\mathcal{C}(B)$ must be Noetherian as well. Hence Proposition 5 implies that $B$ is finite dimensional.

It follows from Lemma 9 that both $\mathcal{C}(A)$ and $\mathcal{C}(B)$ have a rank function assigning to each element its dimension. By Lemma 7 the rank function is unique, hence it follows from Lemma 8 that $\operatorname{dim}(\phi(C))=\operatorname{dim}(C)$ for each $C \in \mathcal{C}(A)$. Therefore, we can reconstruct the dimensions of elements of $\mathcal{C}(A)$ and $\mathcal{C}(B)$, and the dimension is preverved by $\phi$. 
By the Artin-Wedderburn Theorem, there are unique $k, k^{\prime} \in \mathbb{N}$ and unique $\left\{n_{i}\right\}_{i=1}^{n},\left\{n_{i}^{\prime}\right\}_{i=1}^{k^{\prime}}$ with $n_{i}, n_{i}^{\prime} \in \mathbb{N}$ such that

$$
\begin{aligned}
& A \cong \bigoplus_{i=1}^{k} \mathrm{M}_{n_{i}}(\mathbb{C}) ; \\
& B \cong \bigoplus_{i=1}^{k^{\prime}} \mathrm{M}_{n_{i}^{\prime}}(\mathbb{C}) .
\end{aligned}
$$

Without loss of generality, we may assume that the $n_{i}$ and $n_{i}^{\prime}$ form an descending (but not necessarily strictly descending) finite sequence.

By Lemma 13, we have $Z(A)=\bigcap \max \mathcal{C}(A)$ and $\bigcap \max \mathcal{C}(B)=Z(B)$. Since the intersection is the infimum operation in $\mathcal{C}(A)$ and $\mathcal{C}(B)$, and order isomorphism preserve both infima and maximal elements, we find that $\phi(Z(A))=Z(B)$, so $\operatorname{dim}(Z(A))=\operatorname{dim}(Z(B))$. Using Lemma 14, we find that $Z(A)=\bigoplus_{i=1}^{n} Z\left(\mathrm{M}_{n_{i}}(\mathbb{C})\right)$, and since the dimension of the center of a matrix algebra is 1 , we find that $\operatorname{dim} Z(A)=k$. In the same way, we find that $\operatorname{dim} Z(B)=k^{\prime}$, so we must have $k=k^{\prime}$,

Let $M \in \max \mathcal{C}(A)$. Then $\phi(M)$ is a maximal element of $\mathcal{C}(B)$, and $\phi$ restricts to an order isomorphism $[Z(A), M] \rightarrow[Z(B), \phi(M)]$ since $\phi(Z(A))=Z(B)$. By Proposition 7, we obtain an order isomorphism

$$
\prod_{i=1}^{k} \mathcal{C}\left(\mathbb{C}^{n_{i}}\right) \cong \prod_{i=1}^{k} \mathcal{C}\left(\mathbb{C}^{n_{i}^{\prime}}\right) .
$$

It is possible that for some $i$ we have $n_{i}=1$, in which case we have $\mathcal{C}\left(\mathbb{C}^{n_{i}}\right)=\mathbf{1}$. Since we assumed that $\left\{n_{i}\right\}_{i=1}^{n}$ is a descending sequence, there is a greatest number $r$ below $k$ such that $n_{r} \neq 1$. Likewise, let $s$ be the greatest number such that $n_{s}^{\prime} \neq 1$. Then we obtain an order isomorphism

$$
\prod_{i=1}^{r} \mathcal{C}\left(\mathbb{C}^{n_{i}}\right) \cong \prod_{i=1}^{s} \mathcal{C}\left(\mathbb{C}^{n_{i}^{\prime}}\right) .
$$

By Proposition 9 and Corollary 1, we now find $r=s$, and there is a permutation $\pi$ : $\{1, \ldots, r\} \rightarrow\{1, \ldots, r\}$ such that $\mathcal{C}\left(\mathbb{C}^{n_{i}}\right) \cong \mathcal{C}\left(\mathbb{C}^{n_{\pi(i)}^{\prime}}\right)$ for each $i \in\{1, \ldots, r\}$. Let $\psi_{i}$ : $\mathcal{C}\left(\mathbb{C}^{n_{i}}\right) \rightarrow \mathcal{C}\left(\mathbb{C}_{\pi(i)}^{n_{\pi}^{\prime}}\right)$ be the accompanying order isomorphism. Lemma 9 assures that the function assigning to each element of $\mathcal{C}\left(\mathbb{C}^{n_{i}}\right)$ its dimension is a rank function, and similarly the dimension function is a rank function for $\mathcal{C}\left(\mathbb{C}^{n_{\pi(i)}^{\prime}}\right)$. By Lemma 8 , we find that $\operatorname{dim}(C)=$ $\operatorname{dim}\left(\psi_{i}(C)\right)$ for each $C \in \mathcal{C}\left(\mathbb{C}^{n_{i}}\right)$. Hence

$$
n_{i}=\operatorname{dim}\left(\mathbb{C}^{n_{i}}\right)=\operatorname{dim}\left(\psi_{i}\left(\mathbb{C}^{n_{i}}\right)\right)=\operatorname{dim}\left(\mathbb{C}^{n_{\pi(i)}^{\prime}}\right)=n_{\pi(i)}^{\prime},
$$

where the fact that order isomorphisms map greatest elements to greatest elements is used in the third equality.

By definition of $r$, we must have $n_{i}=n_{i}^{\prime}=1$ for all $i \geq r$. Hence we can extend $\pi$ to a permutation $\{1, \ldots, k\} \rightarrow\{1, \ldots, k\}$ by setting $\pi(i)=i$ for each $i \geq r$. Hence $k=k^{\prime}$ and $\left\{n_{1}, \ldots, n_{k}\right\}$ and $\left\{n_{1}^{\prime}, \ldots, n_{k}^{\prime}\right\}$ are the same sets up to permutation. We conclude that $A$ and $B$ must be *-isomorphic.

We note that since the class of all finite-dimensional $\mathrm{C}^{*}$-algebras and the class of all finite-dimensional von Neumann algebras are the same, a similar statement holds for the functor $\mathcal{V}$ assigning to a von Neumann algebra $M$ the poset $\mathcal{V}(M)$ of its commutative von 
Neumann subalgebras. Thus if $M$ and $N$ are von Neumann algebras such that $M$ is finitedimensional, then $\mathcal{V}(M) \cong \mathcal{V}(N)$ implies $M \cong N$.

If $A$ is a finite-dimensional $C^{*}$-algebra and $B$ is a $C^{*}$-algebra such that there is an order isomorphism $\phi: \mathcal{C}(A) \rightarrow \mathcal{C}(B)$, then it might be the case that even though $A$ and $B$ are *-isomorphic, we have $\phi=\mathcal{C}(f)$ for more than one *-isomorphism $f: A \rightarrow B$. For instance, let $A=B=\mathbb{C}^{2}$. Let $f: \mathbb{C}^{2} \rightarrow \mathbb{C}^{2}$ be given by $f(\langle a, b\rangle)=\langle b, a\rangle$. Then both $\mathcal{C}(f)=\mathcal{C}\left(1_{\mathbb{C}^{2}}\right)$.

It might even be the case that $\phi \neq \mathcal{C}(f)$ for each ${ }^{*}$-isomorphism $f: A \rightarrow B$. For instance, let $A=B=\mathrm{M}_{2}(\mathbb{C})$. Then

$$
\mathcal{C}\left(\mathrm{M}_{2}(\mathbb{C})\right)=\left\{\mathbb{C} 1_{\mathrm{M}_{2}(\mathbb{C})}\right\} \cup\left\{u D_{2} u^{*}: u \in \mathrm{SU}(2)\right\},
$$

where $D_{2}=\left\{\operatorname{diag}\left(\lambda_{1}, \lambda_{2}\right): \lambda_{1}, \lambda_{2} \in \mathbb{C}\right\}$. Furthermore, one can show that if

$f: \mathrm{M}_{2}(\mathbb{C}) \rightarrow \mathrm{M}_{2}(\mathbb{C})$ is a *-isomorphism, then $f$ is of the form $a \mapsto u a u^{-1}$ for some $u \in$ $\mathrm{U}(2)$ [1, Theorem 4.27]. Hence $\mathcal{C}(f): \mathcal{C}\left(\mathrm{M}_{2}(\mathbb{C})\right) \rightarrow \mathcal{C}\left(\mathrm{M}_{2}(\mathbb{C})\right)$ is given by $C \mapsto u C u^{*}$ for some $u \in \mathrm{U}(2)$.

Choose $v \in \mathrm{U}(2)$ such that $D_{2} \neq v D_{2} v^{*}$, and let $\phi: \mathcal{C}\left(\mathrm{M}_{2}(\mathbb{C})\right) \rightarrow \mathcal{C}\left(\mathrm{M}_{2}(\mathbb{C})\right)$ be defined by $\phi\left(D_{2}\right)=v D_{2} v^{*}, \phi\left(v D_{2} v^{*}\right)=D_{2}$, and $\phi(C)=C$ for all other $C \in \mathcal{C}\left(\mathrm{M}_{2}(\mathbb{C})\right)$. Then $\phi$ is clearly an order isomorphism. However, $\phi \neq \mathcal{C}(f)$ for each *-isomorphism $f: \mathrm{M}_{2}(\mathbb{C}) \rightarrow \mathrm{M}_{2}(\mathbb{C})$.

\section{Outlook}

We have shown that $\mathcal{C}(A)$ is a complete invariant for finite-dimensional $\mathrm{C}^{*}$-algebras, whereas Mendivil and Hamhalter showed that $\mathcal{C}(A)$ completely determine commutative $\mathrm{C}^{*}$-algebras. The question is whether there are more classes of $\mathrm{C}^{*}$-algebras which can be classified by $\mathcal{C}(A)$. An interesting class might be that of $A F$-algebras, i.e., $\mathrm{C}^{*}$-algebras $A$ that can be approximated by finite-dimensional $C^{*}$-algebras.

Usually one considers only separable AF-algebras, which are $\mathrm{C}^{*}$-algebras $A$ such that $A=\overline{\bigcup_{i=1}^{\infty} A_{i}}$, where $A_{1} \subseteq A_{2} \subseteq \ldots$ is an ascending chain of finite-dimensional $\mathrm{C}^{*}$ subalgebras of $A$. It is well known that this class of AF-algebras can be classified by Bratteli diagrams [5] and by K-theory [13].

One could also look at $C^{*}$-algebras $A$ such that $A=\overline{\cup \mathcal{D}}$ for some directed set $\mathcal{D}$ consisting of finite-dimensional $\mathrm{C}^{*}$-subalgebras of $A$. In this case $A$ need not be separable, and therefore $\mathrm{C}^{*}$-algebras of these form are called non-separable AF-algebras. It turns out that neither Bratteli diagrams nor K-theory can completely classify this class of $\mathrm{C}^{*}$-algebras $[14,29]$. However, as one might have noticed, the framework of $\mathcal{C}(A)$ might be suitable in order to classify non-separable AF-algebras if one compares the definition of non-separable AF-algebras with the content of Proposition 1. If this is indeed the case, then $\mathcal{C}(A)$ might be an interesting alternative for K-theory.

Since $\mathcal{C}(A)$ is a dcpo, and domain theory (see for instance [15]) deals with various properties of dcpos, a first step might be the study of the domain-theoretical properties of $\mathcal{C}(A)$. For details, we refer to [24]. It might be interesting to compare the domain-theoretical properties of $\mathcal{C}(A)$ with those of $\mathcal{V}(M)$, the poset $\mathcal{V}(M)$ of commutative von Neumann subalgebras of a von Neumann algebra $M$. For the von Neumann case, we refer to [11].

It might be interesting to look at non-unital $\mathrm{C}^{*}$-algebras as well. The reason why we did not consider non-unital $\mathrm{C}^{*}$-algebras lies within quantum toposophy, from which this research evolved. In quantum toposophy one is forced to work constructively; and whereas constructive Gel'fand duality for unital commutative $C^{*}$-algebras holds (see for instance 
[2] and [8]), it was not known yet whether the non-unital version holds as well. However, Henry recently proved a non-unital version of constructive Gel'fand duality [20], which suggests that non-unital $\mathrm{C} *$-algebras can be incorporated within quantum toposophy as well.

In the non-unital case one could proceed as follows. If CStar denotes the category of $\mathrm{C}^{*}$ algebras with $*$-homomorphisms as morphisms, we can define the functor $\mathcal{C}_{0}:$ CStar $\rightarrow$ Poset as follows. Given a $\mathrm{C}^{*}$-algebra $A$, we denote the poset of commutative $\mathrm{C}^{*}$-algebras by $\mathcal{C}_{0}(A)$, and if $f: A \rightarrow B$ is a ${ }^{*}$-homomorphism, $\mathcal{C}_{0}(f): \mathcal{C}_{0}(A) \rightarrow \mathcal{C}_{0}(B)$ is defined by $C \mapsto f[C]$. The functor $\mathcal{C}_{0}$ shares some properties with $\mathcal{C}$, for instance Proposition 2 holds as well if we replace $\mathcal{C}$ by $\mathcal{C}_{0}$. It is even the case that we can describe injectivity of a *homomorphism $f: A \rightarrow B$ completely in order theoretic properties of $\mathcal{C}_{0}(f)$. This is possible, since $\mathbf{0}$, the $\mathrm{C}^{*}$-algebra consisting of only one element 0 , is always an element of $\mathcal{C}_{0}(A)$. Hence $f: A \rightarrow B$ is injective if and only if $\mathcal{C}_{0}(f): \mathcal{C}_{0}(A) \rightarrow \mathcal{C}_{0}(B)$ has an upper adjoint $\mathcal{C}_{0}(f)_{*}: \mathcal{C}_{0}(B) \rightarrow \mathcal{C}_{0}(A)$ such that $\mathcal{C}_{0}(f)_{*}(\mathbf{0})=\mathbf{0}$. The latter equality translates to $f^{-1}[\{0\}]=\{0\}$, which exactly states that $f$ is injective.

We expect that Theorem 1 holds as well if we replace $\mathcal{C}$ by $\mathcal{C}_{0}$. Some minor details in the proofs must be adjusted, but we expect that most lemmas still hold, since each finitedimensional $\mathrm{C}^{*}$-algebra $A$ is automatically unital, hence $\mathcal{C}(A)$ can be regarded as subposet of $\mathcal{C}_{0}(A)$.

However, it might be difficult to prove a non-unital version of Mendivil and Hamhalter's theorem to the effect that $\mathcal{C}_{0}(A)$ determines a commutative $\mathrm{C}^{*}$-algebra $A$ up to *-isomorphism, since it is desirable that we can identify $\mathrm{C}^{*}$-ideals of $A$ as elements of $\mathcal{C}_{0}(A)$ in order to reconstruct $A$, and it is not clear how to make this identification. This is already visible if we consider $\mathcal{C}_{0}\left(\mathbb{C}^{2}\right)=\left\{\mathbf{0}, C_{1}, C_{2}, C_{3}, \mathbb{C}^{2}\right\}$, where

$$
\begin{aligned}
& C_{1}=\{\langle\mu, 0\rangle: \mu \in \mathbb{C}\}, \\
& C_{2}=\{\langle 0, v\rangle: v \in \mathbb{C}\} \\
& C_{3}=\{\langle\lambda, \lambda\rangle: \lambda \in \mathbb{C}\} .
\end{aligned}
$$

The least element and the greatest element of $\mathcal{C}_{0}\left(\mathbb{C}^{2}\right)$ are $\mathbf{0}$ and $\mathbb{C}^{2}$, respectively, and $C_{1}, C_{2}, C_{3}$ are mutually incomparable. Here $C_{1}$ and $C_{2}$ are the only elements that correspond to ideals of $\mathbb{C}^{2}$, but it is not possible to distinguish them from $C_{3}$ in an order theoretical way.

Thus $\mathcal{C}_{0}(A)$ has some advantages as well as disadvantages with respect to $\mathcal{C}(A)$. If $A$ is unital, it could be useful to consider both posets at the same time. In this case, $\mathcal{C}(A)$ can be considered a subposet of $\mathcal{C}_{0}(A)$. It might be interesting to remark that in quantum toposophy, a pair $(\mathcal{C}, \mathcal{D})$ of a poset $\mathcal{C}$ and a subposet $\mathcal{D}$ of $\mathcal{C}$ exactly corresponds to a site $(\mathcal{C}, J)$, i.e., a poset $\mathcal{C}$ equipped with a Grothendieck topology, such that the category $\operatorname{Sh}(\mathcal{C}, J)$ of $J$-sheaves is equivalent to $\operatorname{Sets}^{\mathcal{D}^{\text {op }}}$. Hence if $A$ is unital, then the pair $\left(\mathcal{C}_{0}(A), \mathcal{C}(A)\right)$ corresponds to a site $\left(\mathcal{C}_{0}(A), J\right)$ such that $\operatorname{Sh}\left(\mathcal{C}_{0}(A), J\right) \cong \operatorname{Sets}^{\mathcal{C}(A)^{\text {op }}}$. Since one usually studies the topos Sets ${ }^{\mathcal{C}(A)^{\mathrm{op}}}$, it follows that one can integrate $\mathcal{C}_{0}(A)$ in an elegant way in the usual framework of quantum toposophy. For details on Grothendieck topologies and sheaves on posets, we refer to [31].

Acknowledgments The author would like to thank Jonathan Farley, Chris Heunen, Klaas Landsman, Frank Roumen and Sander Wolters for their comments and suggestions. This research has been financially supported by the Netherlands Organisation for Scientific Research (NWO) under TOP-GO grant no. 613.001.013 (The logic of composite quantum systems). 
Open Access This article is distributed under the terms of the Creative Commons Attribution 4.0 International License (http://creativecommons.org/licenses/by/4.0/), which permits unrestricted use, distribution, and reproduction in any medium, provided you give appropriate credit to the original author(s) and the source, provide a link to the Creative Commons license, and indicate if changes were made.

\section{Appendix: Order-Theoretical Notions}

We recall some definitions in order theory and refer to [12] for a detailed exposition.

A poset $(\mathcal{C}, \leq)$ is a set $\mathcal{C}$ equipped with a (partial) order $\leq$. That is, $\leq$ is a binary relation, which is reflexive, antisymmetric and transitive. We often write $\mathcal{C}$ instead of $(\mathcal{C}, \leq)$ if it is clear which order is used. A poset $\mathcal{C}$ becomes a category if we consider its elements as objects, and taking a unique morphism $C \rightarrow D$ if and only if $C \leq D$ for each $C, D \in \mathcal{D}$.

If either $B \leq C$ or $C \leq B$ for each $B, C \in \mathcal{C}$, we say that $\leq$ is a linear order, and we call $\mathcal{C}$ a linearly ordered set. A linearly ordered subposet of a poset is called a chain. Given a poset $\mathcal{C}$ with order $\leq$, we define the opposite poset $\mathcal{C}^{\text {op }}$ as the poset with the same underlying set $\mathcal{C}$, but where $B \leq C$ if and only if $C \leq B$ in the original order.

Let $\mathcal{D} \subseteq \mathcal{C}$ be a subset. Then $\mathcal{D}$ is called an upper set or an up-set if $C \in \mathcal{D}$ and $D \geq C$ implies $D \in \mathcal{D}$ for each $C, D \in \mathcal{C}$; a lower set or an down-set if $C \in \mathcal{D}$ and $D \leq C$ implies $D \in \mathcal{D}$ for each $C, D \in \mathcal{C}$; directed if for each $D_{1}, D_{2} \in \mathcal{D}$ there is a $D_{3} \in \mathcal{D}$ such that $D_{1}, D_{2} \leq D_{3}$; and filtered if for each $D_{1}, D_{2} \in \mathcal{D}$ there is a $D_{3} \in \mathcal{D}$ such that $D_{1}, D_{2} \geq D_{3}$.

If $C \in \mathcal{C}$, we define the up-set and down-set generated by $C$ by $\uparrow C=\{B \in \mathcal{C}: B \geq C\}$ and $\downarrow C=\{B \in \mathcal{C}: B \leq C\}$, respectively. We can define the up-set generated by a subset $\mathcal{D}$ of $\mathcal{C}$ by $\uparrow \mathcal{D}=\bigcup_{D \in \mathcal{D}} \uparrow D$. Similarly, we define the down-set generated by $\mathcal{D}$ by $\downarrow \mathcal{D}=\bigcup_{D \in \mathcal{D}} \downarrow D$. If $B, C \in \mathcal{C}$, then the set $\{D \in \mathcal{C}: B \leq D \leq C\}$ is called the interval between $B$ and $C$, and is denoted by $[B, C]$. Notice that $[B, C]=\uparrow B \cap \downarrow C$. If $[B, C]=\{B, C\}$, then we say that $C$ covers an element $B$, or that $B$ is covered by $C$.

Let $\mathcal{D} \subseteq \mathcal{C}$. Then $D \in \mathcal{D}$ is called a maximal element of $\mathcal{D}$ if $\uparrow D \cap \mathcal{D}=\{C\}$; a minimal element of $\mathcal{D}$ if $\downarrow$ arrow $D \cap \mathcal{D}=\{C\}$; a greatest element of $\mathcal{D}$ if $B \leq D$ for each $B \in \mathcal{D}$; and a least element of $\mathcal{D}$ if $B \geq D$ for each $B \in \mathcal{D}$. Greatest and least elements are always unique. If $\mathcal{C}$ itself contains a least and a greatest element, usually denoted by 0 and 1 , respectively, we say that $\mathcal{C}$ is a bounded. The set of all maximal elements of $\mathcal{C}$ is denoted by $\max \mathcal{C}$, whereas $\min \mathcal{C}$ denotes the set of all minimal elements of $\mathcal{C}$.

If $\mathcal{D} \subseteq \mathcal{C}$, then an element $C \in \mathcal{C}$ such that $D \leq C$ for each $D \in \mathcal{D}$ is called an upper bound of $\mathcal{D}$. Similarly, $C$ is called a lower bound of $\mathcal{D}$ if $C \leq D$ for each $D \in \mathcal{D}$. If $\mathcal{D}$ has a least upper bound or supremum $C$, usually called the join of $\mathcal{D}$ if $\mathcal{D}$ is finite, then we write $C=\bigvee \mathcal{D}$. Dually, if $C$ is a greatest lower bound or infimum of $\mathcal{D}$, usually called the meet of $\mathcal{D}$ if $\mathcal{D}$ is finite, then we write $C=\bigwedge \mathcal{D}$. If $\mathcal{D}$ is a two-point set, say $\mathcal{D}=\left\{D_{1}, D_{2}\right\}$, we write $D_{1} \vee D_{2}$ instead of $\bigvee \mathcal{D}$, and $D_{1} \wedge D_{2}$ instead of $\wedge \mathcal{D}$. We say that $D_{1} \vee D_{2}$ and $D_{1} \wedge D_{2}$ are the binary join and binary meet, respectively, of $D_{1}$ and $D_{2}$. If we consider $\mathcal{C}$ as a category, then the join of $\mathcal{D}$ is exactly the same as the coproduct of $\mathcal{D}$, whereas the meet of $\mathcal{D}$ is exactly the product of $\mathcal{D}$.

If $\mathcal{C}$ is a poset such that all binary meets exists, then we call $\mathcal{C}$ a meet-semilattice. If the supremum of all directed subsets of $\mathcal{C}$ exist, we call $\mathcal{C}$ a directed-complete partial order, abbreviated by dcpo. If all binary meets and joins exists, we call $\mathcal{C}$ a lattice. If all arbitrary suprema and infima exist, then we call $\mathcal{C}$ a complete lattice. Notice that a complete lattice $\mathcal{C}$ is automatically bounded, since $\bigvee \mathcal{C}$ is its greatest element, and $\bigwedge \mathcal{C}$ is its least 
element. Moreover, if $\mathcal{C}$ has all arbitrary infima, it is automatically a complete lattice, since the supremum of a subset $\mathcal{D}$ of $\mathcal{C}$ is given by $\bigvee \mathcal{D}=\bigwedge\{C \in \mathcal{C}: D \leq C \forall D \in \mathcal{D}\}$.

Let $\mathcal{C}_{1}, \mathcal{C}_{2}$ be posets and $\phi: \mathcal{C}_{1} \rightarrow \mathcal{C}_{2}$ a map. Then $\phi$ is called an order morphism if $C \leq D$ implies $\phi(C) \leq \phi(D)$ for each $C, D \in \mathcal{C}_{1}$; an embedding of posets if $\phi(C) \leq \phi(D)$ if and only if $C \leq D$ for each $C, D \in \mathcal{C}_{1}$; and an order isomorphism if it is an order morphism such that $\phi \circ \psi=1_{\mathcal{C}_{2}}$ and $\psi \circ \phi=1_{\mathcal{C}_{1}}$ for some order morphism $\phi: \mathcal{C}_{2} \rightarrow \mathcal{C}_{1}$. Here $1_{\mathcal{C}_{i}}: \mathcal{C}_{i} \rightarrow \mathcal{C}_{i}$ is the identity order morphism. If $\phi$ is an order morphism and there is an order morphism $\psi: \mathcal{C}_{2} \rightarrow \mathcal{C}_{1}$ such that for each $C_{1} \in \mathcal{C}_{1}$ and $C_{2} \in \mathcal{C}_{2}$ we have $\phi\left(C_{1}\right) \leq C_{2}$ if and only if $C_{1} \leq \psi\left(C_{2}\right)$, we say that $\psi$ is the upper adjoint of $\phi$, and $\phi$ the lower adjoint of $\psi$. Clearly an embedding of posets $\phi$ is injective, but the converse does not always hold. Moreover, a map $\phi: \mathcal{C}_{1} \rightarrow \mathcal{C}_{2}$ is an order isomorphism if and only if it is a surjective order embedding. If we consider $\mathcal{C}_{1}$ and $\mathcal{C}_{2}$ as categories, then the upper adjoint is exactly the same as a right adjoint. If $\mathcal{C}_{1}$ and $\mathcal{C}_{2}$ are both lattices, then $\phi$ is called a lattice morphism if $\phi(C \wedge D)=\phi(C) \wedge \phi(D)$ and $\phi(C \vee D)=\phi(C) \vee \phi(D)$ for each $C, D \in \mathcal{C}_{1}$. If $\phi$ is bijective, then $\phi$ is called a lattice isomorphism. A lattice morphism $\phi: \mathcal{C}_{1} \rightarrow \mathcal{C}_{2}$ is automatically an order morphism. An order isomorphism between lattices is automatically a lattice isomorphism.

Let $\mathcal{C}_{1}, \ldots, \mathcal{C}_{n}$ be posets. Then the cartesian product of the $\mathcal{C}_{i}$ is defined as $\prod_{i=1}^{n} \mathcal{C}_{i}$, sometimes also denoted as $\mathcal{C}_{1} \times \ldots \times \mathcal{C}_{n}$, equipped by the order defined by

$$
\left\langle C_{1}, \ldots, C_{n}\right\rangle \leq\left\langle D_{1}, \ldots, D_{n}\right\rangle
$$

if and only if $C_{i} \leq D_{i}$ for each $i=1, \ldots, n$. If $\mathcal{C}_{i}$ is a lattice for each $i=1, \ldots, n$, then $\prod_{i=1}^{n} \mathcal{C}_{i}$ is a lattice as well.

\section{References}

1. Alfsen, E.M., Schultz, F.W.: State spaces of operator algebras, basic theory. Orientations and C*products, Birkhäuser (2001)

2. Banaschewski, B., Mulvey, C.J.: The spectral theory of commutative $\mathrm{C}^{*}$-algebras: the constructive spectrum. Quaestiones Mathematicae 23(4), 425-464 (2000)

3. van den Berg, B., Heunen, C.: Noncommutativity as a colimit. Appl. Categ. Struct. 20(4), 393-414 (2012)

4. Birkhoff, G.: Lattice theory, vol. XXV. American mathematical society colloquium publication (1967)

5. Bratteli, O.: Inductive limits of finite dimensional $C^{*}$-algebras. Trans. Am. Math. Soc., 171 (1972)

6. Butterfield, J., Isham, C.J.: A topos perspective on the Kochen-Specker theorem: I. Quantum states as generalized valuations. I.t. J. Theor. Phys 37(11), 2669-2733 (1998)

7. Connes, A.: A factor not anti-isomorphic to itself. Ann. of Math 101(3), 536-554 (1962)

8. Coquand, T., Spitters, B.: Constructive gelfand duality for $\mathrm{c}^{*}$-algebras, mathematical proceedings of the Cambridge philosophical society, vol. 147, pp. 323-337. Cambridge University Press (2009)

9. Döring, A., Isham, C.J.: What is a thing?' Topos theory in the foundation of physic. In: Coecke, B. (ed.) New Structures in Physics. Lecture Notes in Physics, Springer (2009)

10. Döring, A., Harding, J.: Abelian subalgebras and the Jordan structure of von Neumann algebras. arXiv:1009.4945v1 (2010)

11. Döring, A., Soares Barbosa, R.: Unsharp values, domains and topo. In: Finster, F., Müller, O., Nardmann, M., Tolksdorf, J., Zeidler, E. (eds.) Quantum Field Theory and Gravity Conceptual and Mathematical Advances in the Search for a Unified Framework, pp. 65-96, Birkhäuser (2012)

12. Davey, B.A., Priestley, H.A.: Introduction to Lattices and Order. Cambridge University Press, New York (2002)

13. Elliott, G.A.: On the classification of inductive limits of sequences of semi-simple finite dimensional algebras. Journal of Algebra 38, 29-44 (1976)

14. Farah, I., Katsura, T.: Nonseparable UHF algebras, II (2013). arXiv:1301.6152v1 
15. Gierz, G., Hofmann, K.H., Keimel, K., Lawson, J.D., Mislove, M.W., Scott, D.S.: Continuous Lattices and Domains. Cambridge University Press (2003)

16. Grätzer, G. General Lattice Theory, 2nd edn. Birkhäuser Verlag AG (2003)

17. Hamhalter, J.: Isomorphisms of ordered structures of abelian $C^{*}$-subalgebras of $C^{*}$-algebras. J. Math. Anal. Appl. 383, 391-399 (2011)

18. Hamhalter, J.: Dye's theorem and Gleason's theorem for AW*-algebras. arXiv:1408.4597v1 (2014)

19. Hartshorne, R.: Algebraic Geometry. Springer-Verlag (1977)

20. Henry, S.: Constructive Gelfand duality for non-unital commutative C*-algebras. arXiv:1412.2009 (2014)

21. Heunen, C.: Categorical quantum models and logics, PhD Thesis, Radboud University Nijmegen. http:// hdl.handle.net/2066/74875 (2009)

22. Heunen, C.: Characterizations of categories of commutative $C^{*}$-algebras. Commun. Math. Phys. 331(1), 215-238 (2014)

23. Heunen, C., Landsman, N.P., Spitters, B.: A topos for algebraic quantum theory. Commun. Math. Phys. 291, 63-110 (2009)

24. Heunen, C., Lindenhovius, A.J.: Domains of commutative $C^{*}$-subalgebras, in preparation

25. Heunen, C., Reyes, M.: Active lattices determine AW*-algebras. J. Math. Anal. Appl. 416, 289-313 (2014)

26. Just, W., Weese, M.: Discovering modern set theory, vol. I. The Basics, AMS (1996)

27. Kadison, R.V., Ringrose, J.R.: Fundamentals of the theory of operator algebras, vol. I. American Mathematical Society, Elementary Theory (1997)

28. Kadison, R.V., Ringrose, J.R.: Fundamentals of the theory of operator algebras, vol. III. American Mathematical Society, Elementary Theory - An Exercise Approach (1991)

29. Katsura, T.: Non-separable AF-algebras. arXiv:math/0504423v1 (2005)

30. Kung, J.P.S., Rota, G.-C., Yan, C.H.: Combinatorics: The Rota Way. Cambridge University Press (2009)

31. Lindenhovius, A.J.: Grothendieck topologies on a poset. arXiv:1405.4408 (2014)

32. Mendivil, F.: Compactifications and function spaces, $\mathrm{PhD}$ thesis, school of mathematics, Georgia Institute of Technology, http://math.acadiau.ca/mendivil/Papers/thesis.pdf (1996)

33. Murphy, G.J.: C*-algebras and operator theory. Academic Press (1990)

34. Phillips, N.C.: A simple separable $C^{*}$-algebra not isomorphic to its opposite algebra. Proc. Am. Math. Soc. 132, 2997-3005 (2004)

35. Phillips, N.C., Viola, M.G.: A simple separable exact $\mathrm{C}^{*}$-algebra not anti-isomorphic to itself. Math. Ann 355, 783-799 (2013)

36. Roman, S.: Lattices and ordered sets. Springer-Verlag (2008)

37. Sachs, D.: Partition and modulated lattices. Pac. J. Math 11(1), 325-345 (1961)

38. Spitters, B.: The space of measurement outcomes as a spectral invariant for non-commutative algebras. Found. Phys. 42(7), 896-908 (2012)

39. Takesaki, M.: Theory of Operator Algebra I. Springer-Verlag (1979)

40. Weaver, N.: Mathematical quantization, Stud. Adv. Math. Chapman Hall/CRC (2001)

41. Wolters, S.A.M.: Quantum Toposophy, PhD Thesis, Radboud University Nijmegen. http://hdl.handle. net/2066/112939 (2003) 